Provided for non-commercial research and education use. Not for reproduction, distribution or commercial use.

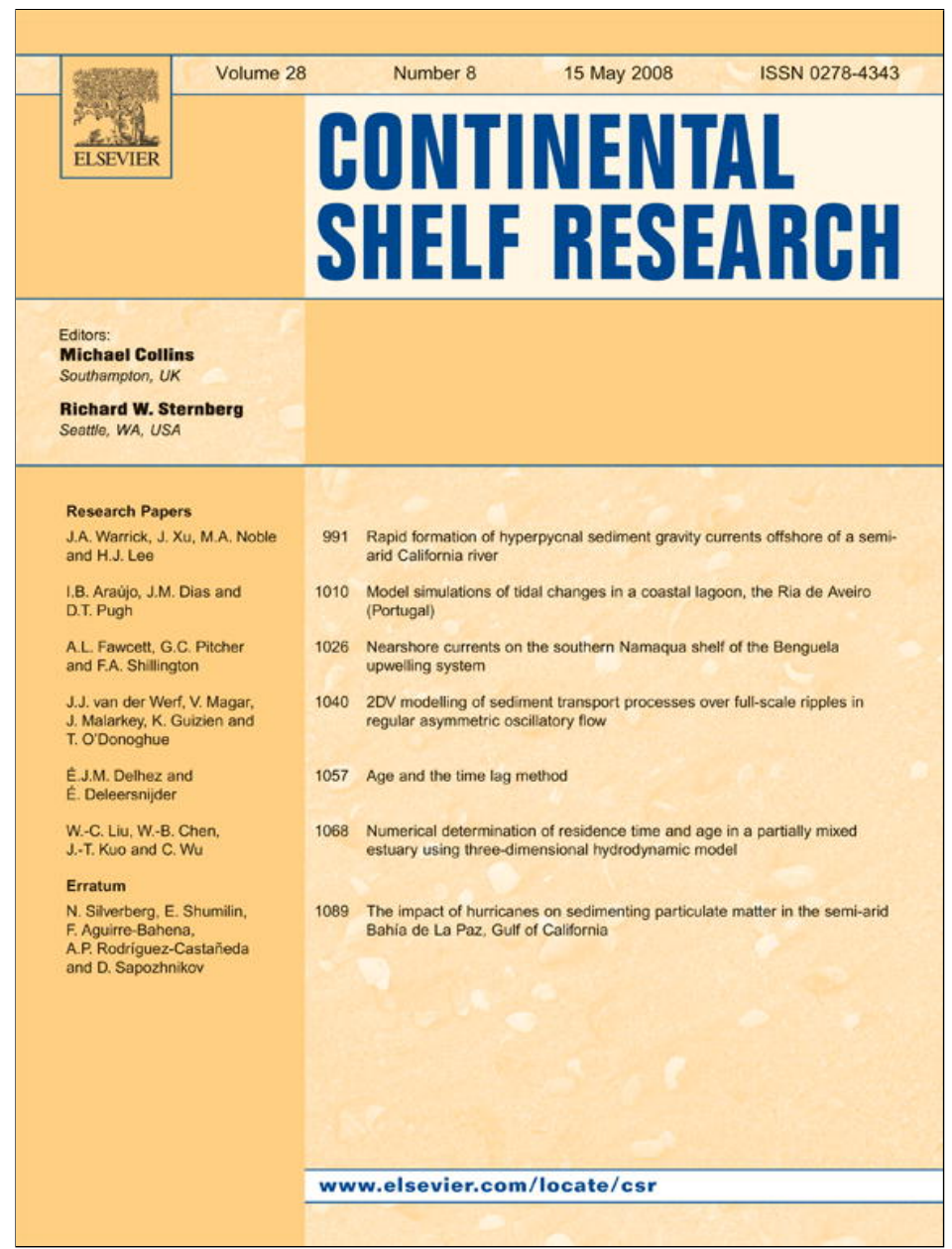

This article appeared in a journal published by Elsevier. The attached copy is furnished to the author for internal non-commercial research and education use, including for instruction at the authors institution and sharing with colleagues.

Other uses, including reproduction and distribution, or selling or licensing copies, or posting to personal, institutional or third party websites are prohibited.

In most cases authors are permitted to post their version of the article (e.g. in Word or Tex form) to their personal website or institutional repository. Authors requiring further information regarding Elsevier's archiving and manuscript policies are encouraged to visit:

http://www.elsevier.com/copyright 


\title{
2DV modelling of sediment transport processes over full-scale ripples in regular asymmetric oscillatory flow
}

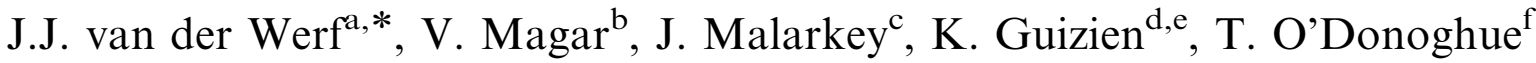 \\ ${ }^{\mathrm{a}}$ Water Engineering and Management, University of Twente, P.O. Box 217, 7500 AE Enschede, The Netherlands \\ ${ }^{\mathrm{b}}$ Centre for Coastal Dynamics and Engineering, University of Plymouth, Drake Circus, Plymouth, PL4 8AA, UK \\ ${ }^{\mathrm{c}}$ School of Ocean Sciences, Bangor University, Anglesey, LL59 5AB, UK \\ ${ }^{\mathrm{d}}$ UPMC Univ Paris 06, UMR 7621, LOBB, UMR 7628, MBCE, Observatoire Océanologique, F-66651 Banyuls/mer, France \\ ${ }^{\mathrm{e}} \mathrm{CNRS}, \mathrm{UMR}$ 7621, LOBB, Observatoire Océanologique, F-66651, Banyuls/mer, France \\ ${ }^{\mathrm{f}}$ Department of Engineering, King's College, University of Aberdeen, Aberdeen, AB24 $3 U E$, Scotland
}

Received 27 March 2007; received in revised form 10 January 2008; accepted 11 February 2008

Available online 26 February 2008

\begin{abstract}
Wave-induced, steep vortex ripples are ubiquitous features in shallow coastal seas and it is therefore important to fully understand and model the sediment transport processes that occur over them. To this end, two two-dimensional vertical (2DV) models have been critically tested against detailed velocity and sediment concentration measurements above mobile ripples in regular asymmetric oscillatory flow. The two models are a $k-\omega$ turbulence-closure model and a discrete-vortex, particle-tracking (DVPT) model, while the data are obtained in the Aberdeen oscillatory flow tunnel (AOFT). The models and the data demonstrate that the time-dependent velocity and suspended sediment concentration above the ripple are dominated by the generation of lee-side vortices and their subsequent ejection at flow reversal. The DVPT model predicts the positions and strengths of the vortices reasonably well, but tends to overpredict the velocity close to the ripple surface. The $k-\omega$ model, on the other hand, underpredicts the height to which the vortices are lifted, but is better able to predict the velocity close to the bed. In terms of the cycle- and ripple-averaged horizontal velocity, both models are able to reproduce the observed offshore flow close to and below the ripple crest and the DVPT model is able to produce the onshore flow higher up. In the vicinity of the vortices, the DVPT model better represents the concentration (because of its better prediction of vorticity). The $k-\omega$ model, on the other hand, better represents the concentration close to the ripple surface and higher up in the flow (because of the better representation of the near-bed flow and background turbulence). The measured and predicted cycle- and ripple-averaged suspended sediment concentrations are in reasonable agreement and demonstrate the expected region of exponential decay. The models are able to reproduce the observed offshore cycle- and ripple-averaged suspended sediment flux from the ripple troughs upwards, and as a result, produce net offshore suspended sediment transport rates that are in reasonable agreement. The net measured offshore suspended transport rate, based on the integration of fluxes, was found to be consistent with the total net offshore transport measured in the tunnel as a whole once the onshore transport resulting from ripple migration was taken into account, as would be expected. This demonstrates the importance of models being able to predict ripple-migration rates. However, at present neither of the models is able to do so.
\end{abstract}

(C) 2008 Elsevier Ltd. All rights reserved.

Keywords: Experimental data; Mathematical models; Oscillatory flow; Sand ripples; Sediment transport

\footnotetext{
${ }^{*}$ Corresponding author. Tel.: + 31534892959 ; fax: + 31534895377 .

E-mail addresses: J.J.vanderWerf@utwente.nl (J.J. van der Werf), Vanesa.Magar@Plymouth.ac.uk (V. Magar), J.Malarkey@bangor.ac.uk (J. Malarkey), Guizien@obs-banyuls.fr (K. Guizien), T.O'Donoghue@eng.abdn.ac.uk (T. O’Donoghue).
}

\section{Introduction}

Except under storm-wave conditions, the shoreface is generally covered with wave-generated ripples. These ripples typically have heights of $0.01-0.1 \mathrm{~m}$ and lengths of $0.1-1.0 \mathrm{~m}$. The ripples that first appear on a flat undisturbed bed under oscillatory flow have been called rollinggrain ripples by Bagnold (1946). Rolling-grain ripples are 
characterised by the absence of flow separation behind the crest as a result of their low steepness, $\eta / \lambda<0.1$, where $\eta$ is the ripple height and $\lambda$ the ripple wavelength. Stable rolling-grain ripples are rarely found in the field or during laboratory experiments, and therefore their actual existence as stable bedform configurations has been questioned (see e.g. Andersen, 1999; Faraci and Foti, 2002). Nevertheless, the presence of rolling-grain ripples results in steady circulation cells forming on both ripple flanks which tend to drive sediment towards the ripple crests thereby increasing the steepness and consequently the strength of the circulation cells. This process continues until the ripple is steep enough for the flow to separate on the lee-side and the equilibrium geometry of the so-called vortex ripple is reached (Sleath, 1984).

Vortex ripples have an important influence not only on the wave boundary layer structure and turbulence intensity near the bed, but also on the sediment transport processes. In a near-bed layer with a thickness of one to two times the ripple height, the flow dynamics are dominated by the coherent, periodic vortex structures that form above the ripple lee-slope. These vortices are generated by the separation at the crest of the flow coming from the stossside of the ripple. The vortices are subsequently ejected into the flow at flow reversal and new vortices are generated on the opposite side of the ripple. This process is repeated each wave half cycle (see e.g. Earnshaw and Greated, 1998; Marin, 2004). Fredsøe et al. (1999) demonstrated that when waves and currents are superimposed this pattern of behaviour persists almost unchanged because of the dominance of these periodic vortex structures near the ripples.

Associated with the generation and ejection of vortices, the suspended sediment above ripples has a well-organised temporal and spatial structure and coherent, sediment-rich, lee vortices represent the primary mechanism for entraining sediment from the bed into the water column (see e.g. Clubb, 2001; Thorne et al., 2003). Above rippled beds, the phase of sediment pick-up from the bed during the wave cycle is thus linked to the phase of vortex shedding. This has potentially important consequences for the net sediment transport rate beneath asymmetrical waves, which can be 'offshore' in spite of the fact that 'onshore' near-bed velocities are stronger (see e.g. Sato, 1987; Clubb, 2001; Van der Werf et al., 2006).

Experimental results are important to understand the complex nature of ripple regime sediment transport. Numerous experimental studies on sediment transport processes over rippled beds in oscillatory flows have been carried out in the past, both in the field (e.g. Vincent and Green, 1990; Green and Black, 1999; Traykovski et al., 1999; Hanes et al., 2001; Doering and Baryla, 2002; Williams et al., 2003; Grasmeijer and Kleinhans, 2004) and in the laboratory (e.g. Bosman, 1982; Faraci, 2001; Thorne et al., 2002; Marin, 2004). The advantages of laboratory experiments are that the conditions are controlled, a high measuring accuracy can be obtained and the total net sediment transport rate can be measured directly. However, due to limitations of the experimental facilities, many laboratory experiments were carried out under smallscale conditions, i.e. relatively short wave periods. One way to overcome this limitation is to perform experiments in a wave tunnel. This has the added advantage of isolating the processes from the effects of the wave's free surface, which aids in the measurement process (e.g. Sato, 1987; Ribberink and Al-Salem, 1994; Clubb, 2001; Van der Werf, 2006).

Especially in the last decade many mathematical models have been developed to compute the sediment transport processes over ripples. Unsteady models, which are the most sophisticated, attempt to represent the detailed physical processes involved in sediment transport by waves and currents over rippled beds. They include one-dimensional models that resolve the vertical- and the timedependent structure of the velocity and sediment concentration field (e.g. Davies and Thorne, 2005) for more practical applications, as well as two- and three-dimensional intra-wave models that represent the processes more completely (e.g. Longuet-Higgins, 1981; Hansen et al., 1994; Perrier, 1996; Watanabe et al., 2003; Eidsvik, 2006). The net sediment transport can be obtained by integrating the cycle- and ripple-averaged sediment flux over the water depth. Intra-wave models can be divided into turbulenceclosure models, discrete-vortex models, large eddy simulation (LES) models and direct numerical simulation (DNS) models. DNS models have been used to represent the full three-dimensional nature of flows over rippled beds (Scandura et al., 2000; Blondeaux et al., 2004), but are currently limited to the laminar regime due to computational capacity restrictions. LES modelling is complex and still in development (Chang and Scotti, 2003, 2006; Watanabe et al., 2003; Tseng and Ferziger, 2004). These two modelling techniques are not included in the present study.

In this paper, we consider two two-dimensional vertical (2DV) sediment transport models: (i) the $k-\omega$ model of Guizien (2005) and (ii) the discrete-vortex, particle-tracking (DVPT) model developed by Malarkey and Davies (2002) and Magar and Davies (2005). These models have not yet been validated against detailed measurements of sediment transport processes in oscillatory flows over full-scale, mobile ripples, mainly due to a lack of such measurements. Recently, a new dataset containing detailed oscillatory flow tunnel measurements of the transport processes over fullscale rippled beds for three different regular asymmetric oscillatory flows in a tunnel has been produced by Van der Werf et al. (2007). In this paper, we use the data from one of these experiments to test the two above-mentioned models. This is the first time that such a detailed intercomparison has been carried out.

This paper is organised as follows. Section 2 gives a short description of the two 2DV models. In Section 3 the experiment is presented. The ripple's periodicity and the sand's settling velocity are also discussed in relation to 
observations and modelling assumptions. In the next two sections the measured and predicted velocities, suspended sediment concentrations, suspended sediment fluxes and net transport rates are compared. Finally, Section 5 presents the conclusions.

\section{2DV Sediment transport models}

The models represent the flow over one ripple crosssection assuming that the along-ripple variation can be neglected and the ripple remains fixed in space and time. They make use of a conformal mapping function to represent the ripple shape (see Malarkey and Davies, 2002). The convention used in this paper is that the $x$-and $y$-axis represent the horizontal (cross-ripple) direction, and the vertical direction, respectively, with corresponding velocity components $u$ and $v$, as shown schematically in Fig. 1. All model quantities are periodic in the horizontal direction over a ripple wavelength $\lambda$. For simplicity, we chose the troughs to correspond to $x= \pm \lambda / 2$ (see Fig. 1). Also, we assume that the presence of the sediment causes no stratification or turbulence damping effects. The models are described below.

\section{1. $k-\omega$ model}

The $k-\omega$ model of Guizien (2005) solves the conservation equations for the Reynolds-averaged velocity, $\mathbf{u}=(u, v)$, the turbulent kinetic energy, $k$, and the specific turbulent kinetic energy dissipation rate, $\omega$, for the flow above ripples using the turbulence-closure proposed by Guizien et al. (2003). By defining the velocity in terms of a stream function $\psi: \mathbf{u}=(\partial \psi / \partial y,-\partial \psi / \partial x)$ and the vorticity, $\Omega$, as $\Omega=\partial u / \partial y-\partial v / \partial x$, the conservation equations can be rewritten as transport equations in $\Omega, k$ and $\omega$. Besides, $\Omega$ and $\psi$ can be related via Poisson's equation. This new system of equations together with one turbulence-closure equation defining the eddy viscosity or momentum diffusivity is solved numerically on an orthogonal, curvilinear grid based on the conformal mapping function with regular horizontal and exponential vertical spacing.

The boundary conditions for $\Omega$ and $\psi$ are derived from those for the velocity: a no-slip condition on the ripple surface and the velocity being equal to the free-stream flow at the top of the model domain. For $k$ and $\omega$ the boundary conditions on the ripple surface are that $k=0$ and that $\omega$ is

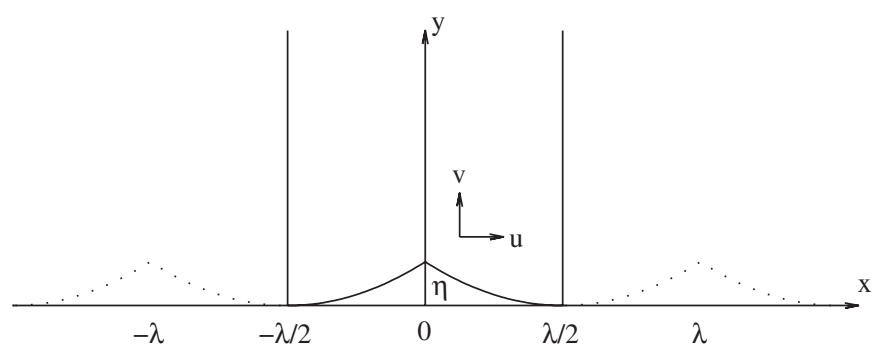

Fig. 1. Definition sketch of the modelling domain for the 2DV models. specified in terms of the cross-ripple friction velocity and the Nikuradse roughness, based on law of the wall arguments (Saffman, 1970). At the top of the model domain, the vertical flux of $k$ and $\omega$ are zero. Finally, as stated earlier, cyclic boundary conditions are applied to all quantities at the lateral boundaries of the computational domain.

With respect to the sediment dynamics, the transport equation for the concentration $c$ is solved in an analogous way to the hydrodynamic equations, with the assumption that the sediment diffusivity is equal to the momentum diffusivity. At the bottom boundary, the concentration is taken as the largest of the reference concentration, $c_{a}$, at two median grain diameters above the bed using the Engelund and Fredsøe (1976) formula and the concentration $c_{b}$ at the same height assuming zero flux perpendicular to the ripple surface. At the top of the model domain, a vertical zero-flux condition is applied. A more complete description of the $k-\omega$ model formulation can be found in Guizien (2005).

\subsection{DVPT model}

The DVPT model of Malarkey and Davies (2002) and Magar and Davies (2005) solves the vorticity transport equation above the ripple directly by representing the vorticity field as a sum of a large number of point vortices. The point vortices are alternately advected by the surrounding flow and given a normally distributed random-walk jump of zero mean and fixed variance, scaled to mimic diffusion by the kinematic viscosity $v$. The computations are carried out in a mapping plane with boundary conditions analogous to those used in the $k-\omega$ model, except that here the no-slip condition for the flow is achieved by introducing new vortices along the ripple surface at every time-step. The velocity and vorticity on the grid are calculated from the point vortices using the cloudin-cell (CIC) method of Christiansen (1973). The flow is phase-ensembled over 30 cycles and the resulting velocity and vorticity are comparable to their Reynolds-averaged counterparts from the $k-\omega$ model. A more complete description of the discrete-vortex part of the model has been given by Malarkey and Davies (2002).

The concentration transport equation is solved by an analogous method to the vorticity transport equation using a particle-tracking method (see Hansen et al., 1994; Perrier, 1996) in a curvilinear coordinate system corresponding to the mapping plane. Sediment particles are continually released from the crest and advected by the phaseensembled velocity, subject to a constant settling velocity and diffused via random-walk jumps with zero mean and fixed variance scaled to mimic the microscopic mixing coefficient $\varepsilon$. The size of $\varepsilon$ was chosen as $400 v$, as this value was found to produce a cycle- and ripple-averaged concentration profile that decayed exponentially with height, as expected from observations (e.g. Thorne et al., 2003). At its release, each 'notional' particle is assigned a 
mass based on an excess shear stress criterion (Nielsen, 1992). The shear stress is calculated using Fredsøe's (1984) boundary layer theory. At the ripple surface, particles having hit the bed through advection and settling are eliminated from the computations, while those having hit the bed through a random jump are reintroduced into the suspension layer. The concentration on the grid is determined by the CIC method and phase-ensembling is over 15 cycles. A more complete description of the particletracking part of the model can be found in Magar and Davies (2005).

\section{The Mr5b63 experiment}

Below follows a concise description of the Mr5b63 experiment. A more complete one can be found in Van der Werf et al. (2007).

\subsection{Experimental facility}

The Mr5b63 experiment was performed by Van der Werf et al. (2007) in the Aberdeen oscillatory flow tunnel (AOFT). The AOFT is a large laboratory facility in which near-bed horizontal flows, equivalent in period and amplitude to the near-bed horizontal flows induced by full-scale waves, can be generated over sediment beds. The AOFT has an overall length of $16 \mathrm{~m}$ with a $10 \mathrm{~m}$ long, glass-sided rectangular test section, $0.75 \mathrm{~m}$ high and $0.3 \mathrm{~m}$ wide, which has a closed top. The test section was filled with a $0.25 \mathrm{~m}$ thick sediment bed leaving $0.5 \mathrm{~m}$ for the flow.

\subsection{Measurements}

Flow velocities over the ripples were measured using a cross-correlation particle image velocimetry (PIV) system based on a double-pulsed $\mathrm{Nd}-$ Yag laser light sheet and a $1 \mathrm{k} \times 1 \mathrm{k}$ cross-correlation camera. The camera and laser are synchronised and the camera grabs a pair of images, with a $2 \mathrm{~ms}$ time separation, at a rate of approximately $13.2 \mathrm{~Hz}$. For the present experiments, measurements were made using a camera viewing area of $400 \times 400 \mathrm{~mm}$. The image pairs were analysed using a $32 \times 32$ pixel interrogation area with $50 \%$ overlap. The spatial resolution of the resulting velocity field measurement is $6.4 \mathrm{~mm}$ horizontally and vertically and the velocity resolution is $20 \mathrm{~mm} / \mathrm{s}$. In order to minimise the effects of ripple migration and distortion, the PIV-measured velocities were phase-ensembled over five flow cycles. The limit that this comparatively small number of cycles places on the interpretation of the results is discussed later (Section 4.1).

The suspended sediment acted as the seeding agent, and therefore measured velocities are those of the suspended sediment rather than the water itself. The settling-velocity effect was removed from the PIV data by first forcing the velocity data to be periodic in the horizontal direction and then removing the ripple-averaged vertical velocity at each height (as this quantity must be zero at all heights to satisfy continuity). This procedure is explained in more detail in Appendix A. As seen from Fig. A1 in Appendix A, the velocity satisfies better the condition of local tangency at the rippled bed with this correction than without it. The size of the ripple-averaged vertical velocity component that is removed will be returned to in the discussion of the choice of settling velocity. The method for calculating the vorticity from the PIV data is explained in Appendix B.

Ripple dimensions were measured using a laser displacement sensor (LDS) mounted on a positioning carriage. The LDS made point measurements of bed elevation with a $0.05 \mathrm{~mm}$ resolution in the vertical direction. The laser displacement system was used to measure the equilibriumrippled bed. Six parallel profiles were measured, spaced at $40 \mathrm{~mm}$ intervals across the tunnel width, with heights measured every $5 \mathrm{~mm}$ along each profile.

An acoustic backscatter system (ABS) was deployed to measure time-dependent suspended sediment concentrations. The ABS collected backscatter profiles at $128 \mathrm{~Hz}$. The ABS data were block averaged to give backscatter profiles measured at $8 \mathrm{~Hz}$ and these are subsequently converted to high-resolution concentration profiles. The averaging is required, because of the statistical nature of the backscattered signal. The system provides concentration profiles with a $0.005 \mathrm{~m}$ vertical spatial resolution. Calibration was based on concentrations measured by an optical concentration metre (OPCON) and a transverse suction system (TSS). Van der Werf et al. (2007) showed that the ABS- and OPCON-measured time-dependent concentration profiles were very similar, thus demonstrating the consistency of the concentration measurements.

The ABS measured continuously while six entire equilibrium ripples migrated underneath it. The bed level below the ABS was continuously monitored by a sonar ripple profiler. The ABS concentration measurements are accurate within approximately a factor of 2 due to uncertainties in the suspended sand grain size (Chris Vincent, personal communication) with an uncertainty in the vertical position of $\pm 5 \mathrm{~mm}$.

A good approximation of the concentration as a function of time can be found by phase-ensembling over a sufficiently large number of flow cycles. Since the ripples migrate, phase-ensembling over a number of flow cycles also implies bed-averaging. It is assumed that a spatial resolution of $5 \%$ of the ripple length $(20 \mathrm{~mm})$ is acceptable, which corresponds to phase-ensembling the ABS concentrations over 22 flow cycles. This approach is similar to that of Thorne et al. (2003). With this number of flow cycles the typical time-dependent concentration behaviour (number and timing of concentration peaks) is captured. As with the PIV data, the concentration is forced to be periodic over the ripple wavelength.

A TSS was used to measure the time-averaged suspended sediment concentration at five different elevations above the bed. The estimated total (random) error in the measured concentration is $5 \%$, and the uncertainty in vertical position with respect to the ripple surface $\pm 4 \mathrm{~mm}$. 
This estimation does not include uncertainties associated with the ripple size and shape.

The net sediment transport rates were calculated using a mass conservation technique. Given the sediment masses in the traps at both ends of the test section and the volume change derived from the bed profiling system, the net sediment transport rates along the test section were calculated by integrating in from the left-hand and the right-hand boundaries. The calculated net transport rate in (or close to) the middle of the test section was taken as a representative value. Repeated measurements for the same condition show that the (random) error in the measured net transport rate is around $20 \%$.

\subsection{Experimental conditions}

The sediment used for the Mr5b63 experiment was wellsorted with a median grain diameter $D_{50}=0.44 \mathrm{~mm}$ and a grain size distribution as shown in Fig. 2.

The free-stream velocity, $u_{\infty}$ (positive $x$ corresponds to the 'onshore' direction) was based on a wave-tunnel equivalent to near-bed flow beneath Stokes second-order waves, namely

$u_{\infty}=U_{1} \cos (\sigma t-\gamma)+U_{2} \cos (2 \sigma t-2 \gamma)$,

where $t$ is time, $\sigma=2 \pi / T$ is the angular frequency, $T$ is the flow period, $U_{1}$ and $U_{2}$ are the first and second harmonic velocity amplitudes and $\gamma$ is the phase such that $u_{\infty}(0)=0$ :

$\gamma=\arccos \left(\frac{\sqrt{U_{1}^{2}+8 U_{2}^{2}}-U_{1}}{4 U_{2}}\right)$.

Here $T=5.0 \mathrm{~s}, \quad U_{1}=0.54 \mathrm{~m} / \mathrm{s}$ and $U_{2}=0.09 \mathrm{~m} / \mathrm{s}$. This asymmetric oscillatory flow in the tunnel gave rise to a cycle-mean flow that was measured using the PIV system. The ripples for this experiment were highly two-dimensional with height $\eta=0.078 \mathrm{~m}$ and length $\lambda=0.41 \mathrm{~m}$. The ripple size in the central $4 \mathrm{~m}$ of the test section (where the measurements were carried out) was very regular: the relative standard deviation of both the ripple height and length was $13 \%$.

\subsection{Model inputs}

Both models were driven hydrodynamically by the freestream velocity specified in Eqs. (1) and (2) and matched to the cycle- and ripple-averaged velocity as determined by the PIV measurements at a particular height in the same way as is done for $1 \mathrm{DV}$ modelling (e.g. Holmedal and Myrhaug, 2006). The matching height above the ripple trough was taken to be $0.84 \lambda$ since this represented a suitable height outside the wave boundary layer where the velocity shear was zero. The Nikuradse roughness on the ripple surface, $k_{\mathrm{s}}$, was given by the median grain size of the bed sediment $D_{50}, k_{s}=2.5 D_{50}$. In this intercomparison the modellers were also provided with the experimental ripple profile and the settling velocity in suspension. In the interests of minimising the differences in inputs between models, both models used the same ripple profile and settling velocity in suspension. The method of calculating these two inputs is explained in the next two sections.

\subsubsection{The ripple profile}

Both models use the same conformal mapping function to define the orthogonal curvilinear grid. The ripple was forced to be periodic and then fitted to the mapping function (see Malarkey and Davies, 2002) using 16 coefficients. The ripple generated by the mapping function and that measured are depicted in Fig. 3. It is clear that the fitted and the measured ripples are in excellent agreement and that forcing periodicity only results in a slight difference at the ripple troughs.

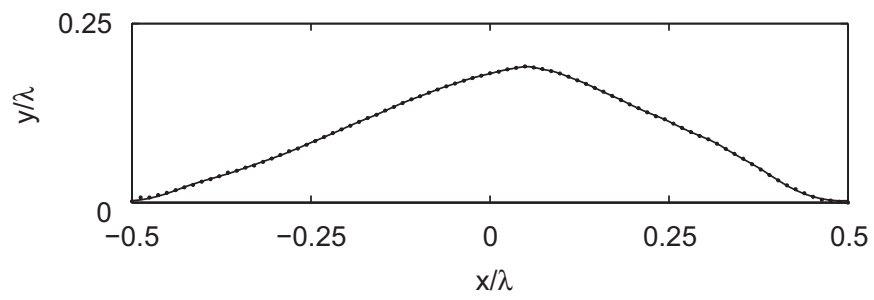

Fig. 3. Comparison of experimental (dots) and fitted (solid line) ripple profile.

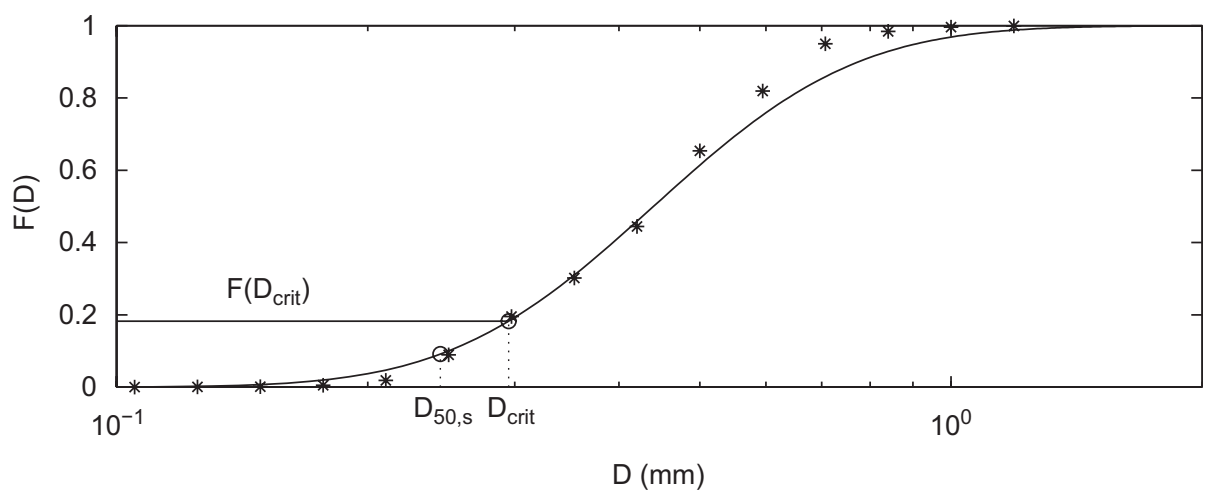

Fig. 2. Grain size distribution for the Mr5b63 experiment (asterisks), showing the maximum critical grain diameter that can get into suspension ( $D_{\text {crit }}$ ), the median grain diameter in suspension $\left(D_{50, \mathrm{~s}}\right)$ (open circles) and a log-normal fit to the lower part of the distribution (solid line) (see Section 3.4.2). 


\subsubsection{The settling velocity}

For modelling purposes it is assumed that the grain size, $D$, is log-normally distributed, such that the cumulative frequency $F(D)$ can be expressed as

$F(D)=0.5\left[1+\operatorname{erf}\left(\frac{\log \left(D / D_{50}\right)}{\sqrt{2} \log \sigma_{g}}\right)\right]$.

Eq. (3) was fitted to the lower end of the distribution using the experimental values of $D_{10}=0.25 \mathrm{~mm}$ and $D_{50}=$ $0.44 \mathrm{~mm}$ to obtain the value of $\sigma_{g}(=1.55)$. It can be seen in Fig. 2, where the log-normal fit is compared to the measured distribution, that Eq. (3) fits the experimental cumulative frequency at the lower end of the grain size distribution reasonably well.

An estimation of the settling velocity, $v_{s}$, can be obtained by calculating the maximum grain size that can get into suspension using Fredsøe and Deigaard's (1992) criterion. A $k-\varepsilon$ model, see for example Malarkey et al. (2003), with the Mr5b63 hydrodynamic conditions and a flat rough bed with $k_{\mathrm{s}}=2.5 D_{50}$, produces a maximum skin friction $u_{* \mathrm{~m}}$ equal to $4.57 \mathrm{~cm} / \mathrm{s}$. Fredsøe and Deigaard's criterion states that the maximum settling velocity, $v_{\mathrm{sc}}$, of the sediment in suspension is $0.8 u_{* \mathrm{~m}}$, which here leads to a maximum settling velocity of $3.65 \mathrm{~cm} / \mathrm{s}$. The maximum settling velocity corresponds to sediment grains with critical grain diameter $D_{\text {crit }}=0.295 \mathrm{~mm}$, as deduced from Hallermeier's (1981) formula. Now, from Eq. (3) it may be found that $F\left(D_{\text {crit }}\right)=0.183$, which means that only $18 \%$ of the grain population can get into suspension. Since $F\left(D_{50, \mathrm{~s}}\right)=$ $0.5 F\left(D_{\text {crit }}\right)$, this implies that $D_{50, \mathrm{~s}}=0.244 \mathrm{~mm}\left(D_{50, \mathrm{~s}}\right.$ and $D_{\text {crit }}$ are shown in Fig. 2). Thus, the (median) settling

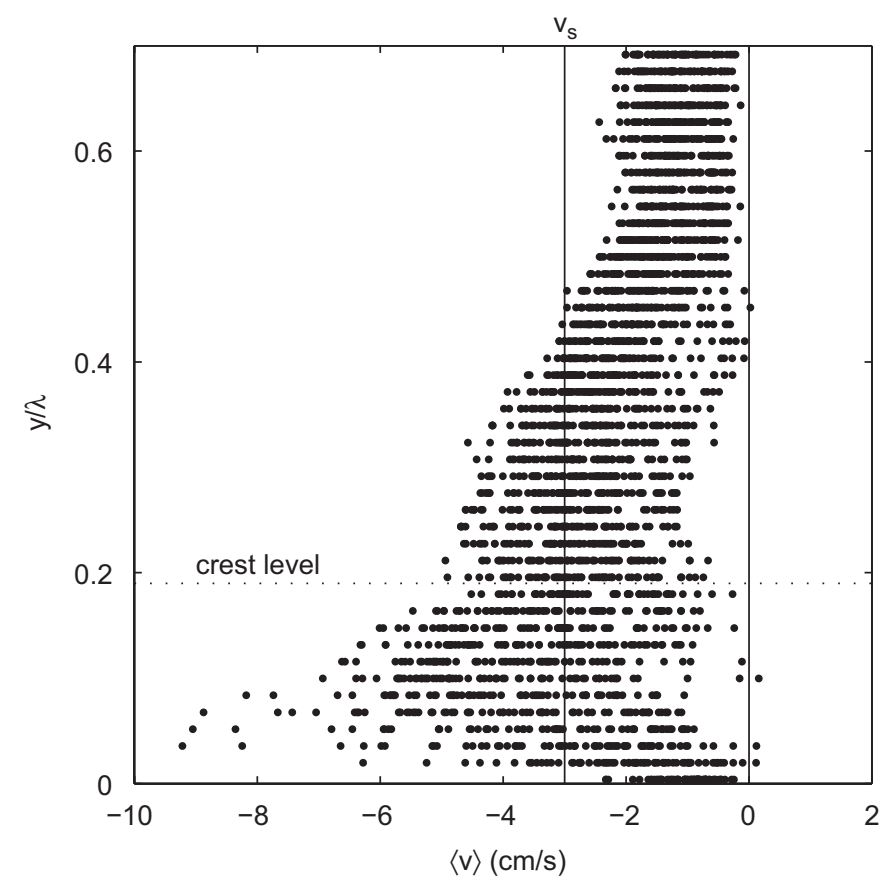

Fig. 4. Comparison of the ripple-averaged vertical velocity from the PIV data and the settling velocity based on Fredsøe and Deigaard's (1992) approach. velocity of the sediment in suspension is $v_{\mathrm{s}}=2.97 \mathrm{~cm} / \mathrm{s}$. It should be pointed out that while the entire sediment population is mobile, only sizes finer than $D_{\text {crit }}=0.295 \mathrm{~mm}$ are suspended and hence all suspended sediment quantities need to be multiplied by $F\left(D_{\text {crit }}\right)=0.183$.

It is interesting to compare this settling velocity estimate with the ripple-averaged vertical velocities, $\langle v\rangle$, which were removed from the PIV data. This comparison is shown in Fig. 4. First of all it can be seen that $\langle v\rangle$ takes values that are consistent with a grain size dependent settling velocity: it is overwhelmingly negative and decreases in magnitude with height above the bed (representing the fining of sediment with height). At each height there is a lot of scatter in $\langle v\rangle$ but it appears to be quite consistent with $v_{\mathrm{s}}$, thereby confirming this choice of settling velocity in the model simulations.

\section{Intercomparison of the hydrodynamics}

\subsection{Vorticity dynamics}

Fig. 5 shows the measured and predicted non-dimensional vorticity $\left(\Omega \lambda / U_{1}\right)$ above the rippled bed at phase angles $\sigma t=0-300^{\circ}$ in steps of $60^{\circ}$. Positive, 'onshore' flow is directed to the right and $\sigma t=0^{\circ}$ corresponds to the offonshore free-stream reversal. Each panel is one ripple wavelength long and one half-ripple wavelength high. Fig. 5 shows vorticity contours predicted by the two models, together with those from the PIV measurements.

At $\sigma t=0^{\circ}$ both models and data clearly show a vortex with negative (anti-clockwise) vorticity, being ejected into the water column as the flow begins to move from left to right. This vortex, labelled ' $F_{0}$ ', was generated in the previous offshore flow half cycle. The proximity of vortex $F_{0}$ to the bed, together with the no-slip condition on the bed, generates shear that results in a counter-rotating strip of vorticity, labelled ' $C$ ', beneath the vortex (see e.g. Malarkey and Davies, 2002). This strip of vorticity is present at phases $0^{\circ}, 120^{\circ}$ and $300^{\circ}$ for both the DVPT model and the data, but only present at phase $120^{\circ}$ for the $k-\omega$ model. If we look at phase $\sigma t=60^{\circ}$, the free-stream is moving onshore and accelerating. Vortex $F_{0}$ has now been advected onshore, and has reached the offshore side of the neighbouring onshore ripple and weakened in strength. By the time the phase reaches $120^{\circ}$, vortex $F_{0}$ appears to be in roughly the same position as at phase $0^{\circ}$ (slightly to the left of the crest) but is now two ripple wavelengths away from its parent ripple. The elevated vortex labelled ' $N_{0}$ ', visible at phases of $0^{\circ}$ and $60^{\circ}$ in the DVPT model and $0^{\circ}$ in the data, is actually the vortex created during the previous wave half cycle on the onshore side of the ripple.

The motion of the vortex $N_{0}$ during the first half cycle depicted in Fig. 5 is in the onshore direction, just as with vortex $F_{0}$, but by $120^{\circ}$ the strength of $N_{0}$ is very small. Moreover, between $\sigma t=0^{\circ}$ and $120^{\circ}$ a new vortex with positive vorticity, labelled ' $N$ ', has been forming on the onshore side of the ripple, starting with the contribution of 

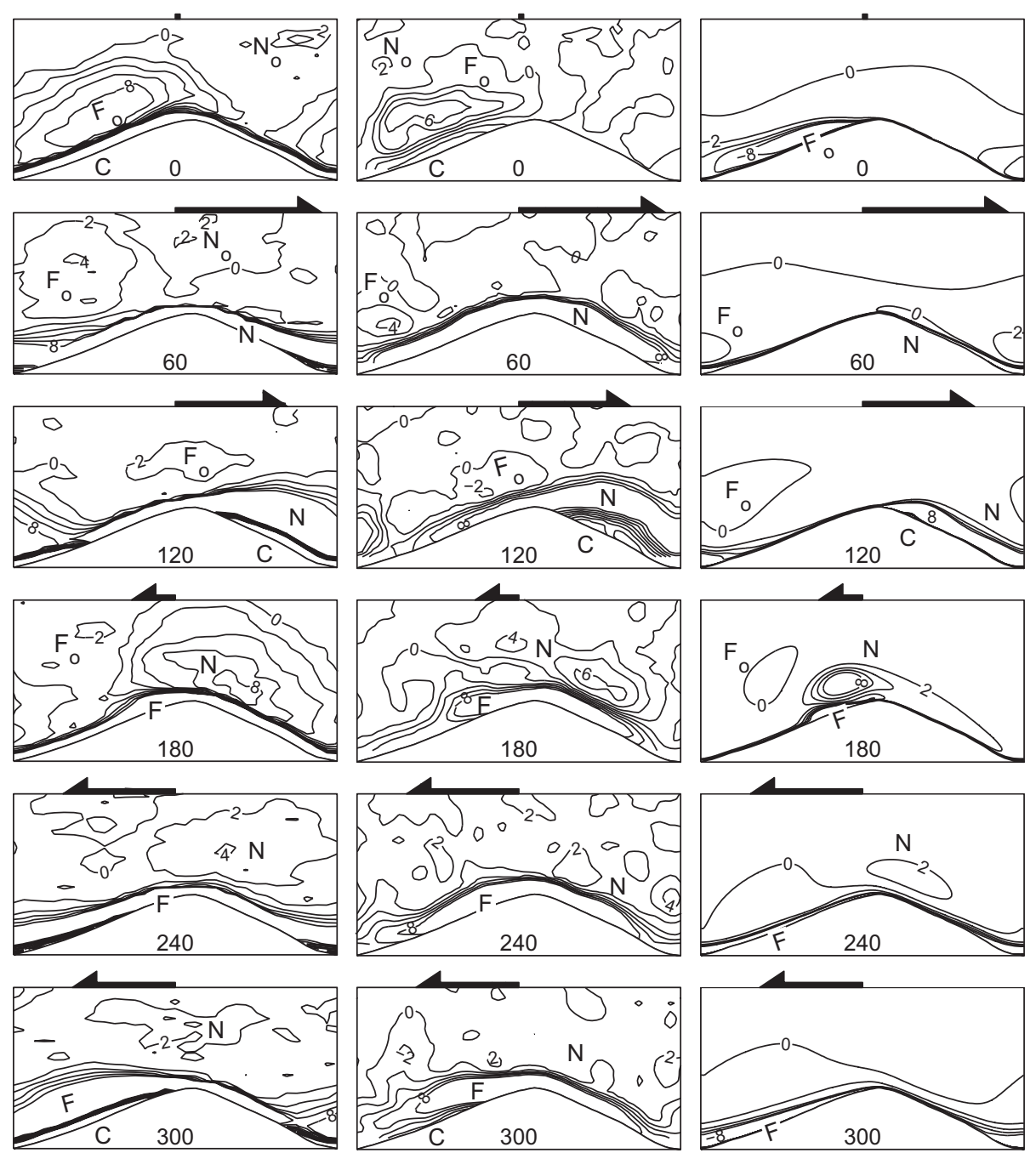

DVPT
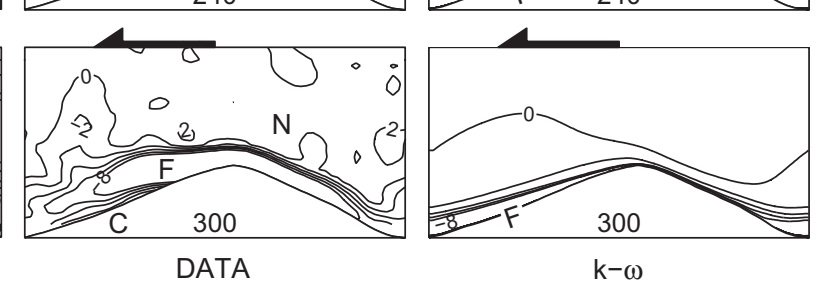

Fig. 5. Non-dimensional vorticity contours, $\Omega \lambda / U_{1}$, (clockwise is positive, spacing $=2$ ) predicted by the DVPT model (left panels), measured in the experiment (middle panels), and predicted by the $k-\omega$ model (right panels) for the phases shown (degrees). $N_{0}$ and $F_{0}$ denote the onshore- and offshoregenerated vortices from the previous wave cycle, $N$ and $F$ denote the onshore- and offshore-generated vortices from the present wave cycle and $\mathrm{C}$ denotes the counter-rotating strip of vorticity. The arrows are proportional to the strength of the free-stream velocity.

the offshore counter-rotating strip of vorticity $C$ at $\sigma t=0^{\circ}$. As the phase advances, vortex $N$ grows as it is in constant contact with the bed.

In general, over phases $0^{\circ}, 60^{\circ}$ and $120^{\circ}$ both models predict the horizontal position of the vortices reasonably well. By the time the phase is $120^{\circ}$ vortex $F_{0}$ in the data is just beginning to break up into more than one region of vorticity. The DVPT model predicts the vertical position, physical size and strength of the vortices better than the $k-\omega$ model. The underprediction by the $k-\omega$ model of the heights reached by the vortices particularly when they are growing is related to the underprediction by this model of the counter-rotating strip of vorticity, which is present in both the DVPT predictions and the experiments. As observed above, the $k-\omega$ model predicts this strip of vorticity only when the phase is $120^{\circ}$. This is possibly due to the boundary condition on the rippled bed: the $k-\omega$ model considers that the vorticity depends explicitly on the second derivative normal to the bed of the stream function and on the value of the Jacobian at the bed. The latter quantity is very sensitive to the steepness of the ripple. Therefore, given that in the experiment analysed here the ripple steepness is quite low, the $k-\omega$ model underpredicts the extent to which the vorticity separates. This is supported by the fact that in Magar et al. (2005), where an experiment with a steeper ripple was studied, the vorticity calculated by the $k-\omega$ model separated much more readily from the bed (see also Fredsøe et al., 1999).

Next, we compare the model predictions and experimental data for phase angles after on-offshore flow reversal, which occurs at $\sigma t=162^{\circ}$. Thus, the first phase after flow reversal is $\sigma t=180^{\circ}$, when the free-stream is moving offshore and the left side of the ripple is now the 
lee-side. Since the flow has reversed, vortex $N$ has been ejected and is now being advected offshore. Given that at $\sigma t=180^{\circ}$ only about $18^{\circ}$ have elapsed since flow reversal, this vortex has not moved very far from its parent ripple, as can be clearly seen in Fig. 5. However, there has been sufficient offshore flow for a new vortex $F$ to start forming on the offshore side of the ripple. It is interesting to note that, in spite of the free-stream velocity asymmetry, the horizontal positions of vortex $F_{0}$ between $0^{\circ}$ and $120^{\circ}$ and vortex $N$ between $180^{\circ}$ and $300^{\circ}$ are the mirror images of one another. This is particularly true for the DVPT model, but less the case for the measurements and even lesser the case for the $k-\omega$ model. Thus in the DVPT model the slower offshore advection is more or less compensated for by considering phase instants which are $18^{\circ}$ ahead in phase, relative to flow reversal, than their onshore counterparts. The free-stream asymmetry, however, does result in a difference in strength between vortices generated in each of the two half flow cycles. It can be seen from both models and the data that the vorticity of vortex $N$ is more diffuse than that of vortex $F_{0}$. In the case of the data this results in the position of vortex $N$ being more ill-defined than the vortex $F_{0}$ once ejected. When the phase is $360^{\circ}$, vortices $N$ and $F$ become $N_{0}$ and $F_{0}$ and the process repeats itself in the next flow cycle.

Finally, it should be noticed that the vorticity contours derived from the velocities measured experimentally appear quite noisy. This is probably related to the fact that the velocities were phase-ensembled for only five cycles, which may not be enough to remove the turbulent component from the velocity field. This is also the reason for the ill-defined nature of the vortex positions, after they have been ejected. However, both the models and experiments reproduce qualitatively well the vortex dynamics above ripples that has been reported in previous studies, both for oscillatory flows (e.g. Malarkey and Davies, 2002) and for surface waves and currents (e.g. Fredsøe et al., 1999). The consistency and uncertainty of the vorticity is discussed further in Appendix B.

\subsection{Cycle-averaged flow field and vorticity contours}

The cycle-averaged flow fields and corresponding vorticity contours are shown in Fig. 6. Cycle-averaging the timevarying flow and vorticity results in two circulation cells, one on either side of the ripple. These cells are mainly the result of the growing vortices, which are almost static when they are forming, and at their strongest just before ejection (Malarkey and Davies, 2002). It can be seen from the vorticity comparisons that the DVPT model predicts the position, strength and extent of the circulation cells reasonably well. The $k-\omega$ model, on the other hand, underpredicts quite substantially the height of the circulation cells and their extent, but predicts their strengths reasonably. These observations are consistent with those made for the time-varying vorticity comparisons.

Both models show an asymmetry in the circulation cells that is consistent with the data, as demonstrated by the relative position of the zero vorticity contour line. However, in the case of the DVPT model there is
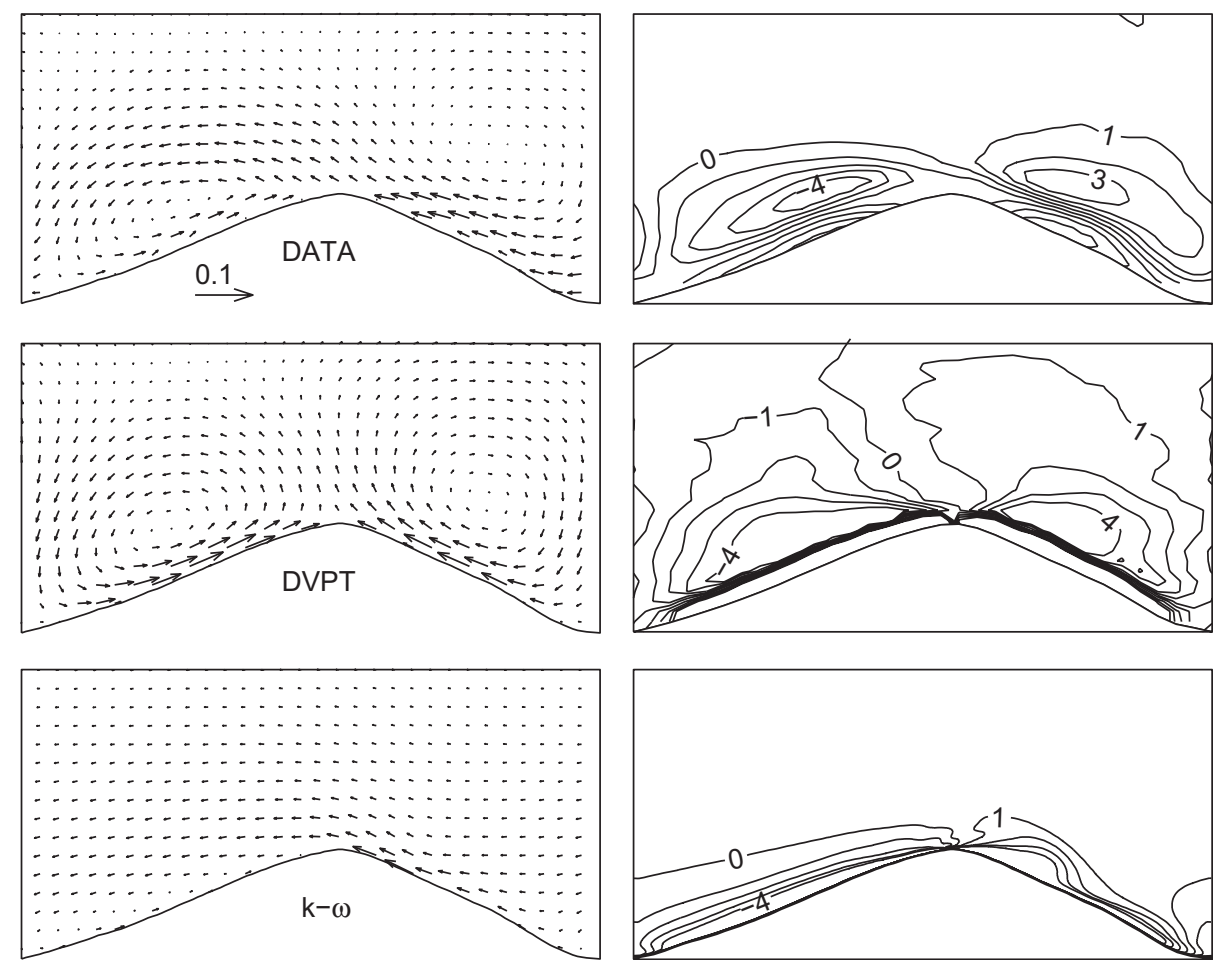

Fig. 6. Cycle-averaged velocity vectors and vorticity contours (non-dimensionalised by $U_{1}$ and $U_{1} / \lambda$, respectively) for the data, the DVPT model, and the $k-\omega$ model (common velocity scale shown in DATA panel). 
insufficient difference in the vertical position of the cells, so that the asymmetry appears weaker. In general however, the DVPT model produces comparable velocities to the data above the ripple but overpredicts the velocity close to it. The $k-\omega$ model, on the other hand, underpredicts the velocity higher above the ripple, but predicts to a better extent the velocity near the ripple. This is probably because only the $k-\omega$ model resolves the boundary layer properly, as it considers the grain roughness at the ripple surface (see Guizien, 2005).

\subsection{Cycle- and ripple-averaged horizontal velocities}

Next, we consider the cycle- and ripple-averaged horizontal velocities above the ripple crest, shown in Fig. 7. As explained in Section 3.4, both models were matched to the cycle- and ripple-averaged experimental velocity at a certain height $(0.84 \lambda$ above the ripple trough) and this can clearly be seen in Fig. 7.

The DVPT model predicts the velocity profile reasonably well, including the correct position of the offshore and onshore maxima. However, the magnitude of the onshore maximum is slightly overpredicted and the magnitude of the offshore maximum is more substantially underpredicted, especially below the crest level. This latter underprediction probably relates to the insufficient difference in the vertical position of the circulation cells (discussed in Section 4.2). The $k-\omega$ model, on the other hand, predicts the velocity close to the ripple better than the DVPT model but fails to capture the onshore maximum because the circulation cells are too close to the bed.

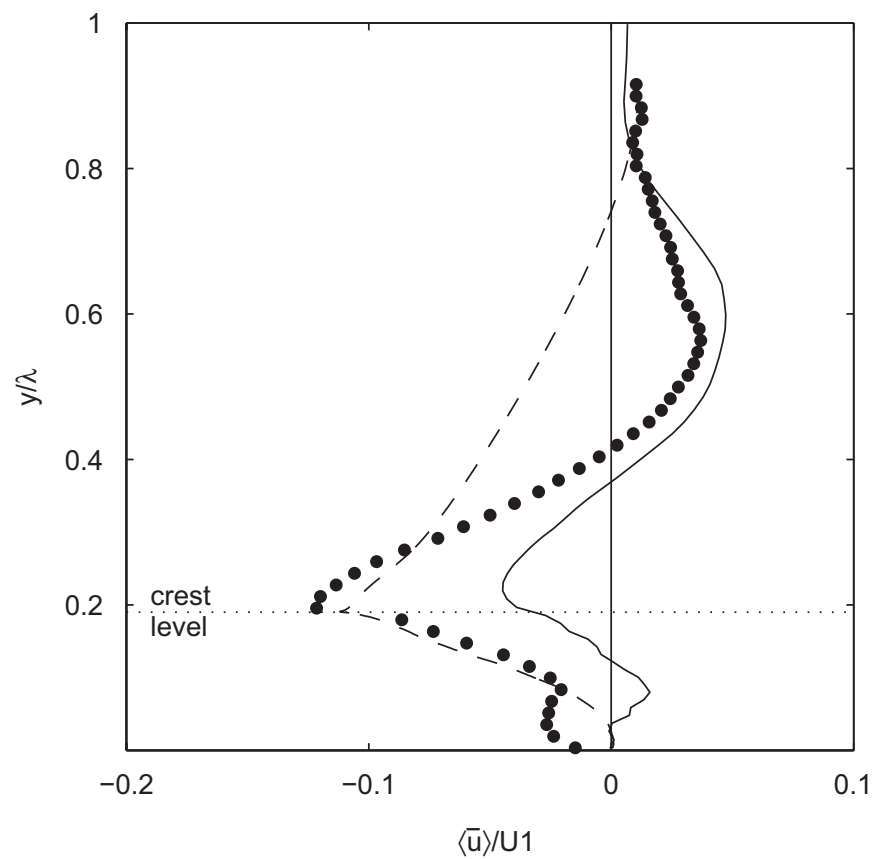

Fig. 7. Cycle- and ripple-averaged horizontal velocities predicted by the DVPT (solid line) and $k-\omega$ (dashed line) models and measured in the experiment (closed circles).

\section{Intercomparison of the suspended sediment dynamics}

\subsection{Time-dependent suspended sediment concentrations}

Fig. 8 shows the measured and predicted suspended sediment concentration contours above the rippled bed at phase angles $\sigma t=0^{\circ}$ to $300^{\circ}$ in steps of $60^{\circ}$. The concentration scale is logarithmic and the same in all the figures; concentrations are in $[\mathrm{g} / \mathrm{l}]$. As with Fig. 5, each panel is one ripple wavelength long and one half-ripple wavelength high. For clarity a minimum concentration of $0.01 \mathrm{~g} / 1$ has been assumed for the models.

In the first instance, we compare the concentration contour plots from the DVPT model (left-hand panels in Fig. 8) to the corresponding vorticity contour plots from that same model, as illustrated in Fig. 5. The comparison of those plots indicates that the positions of the sediment clouds, at each phase, agree reasonably well with the positions of the vortices along and above the ripple surface, at that same phase. Also, there is good correlation between the regions of large vorticity magnitude and the regions of large sediment concentration.

Next, we compare the right-hand panels in Fig. 8 showing the concentration contours predicted by the $k-\omega$ model with the $k-\omega$ model vorticity contours at the corresponding phases (shown in Fig. 5). Again, the sediment clouds seem to follow the vorticity contours quite closely, except at flow reversal. For instance, when the phase is $180^{\circ}$ we would have expected the sediment cloud seen at $120^{\circ}$ to have moved close to the ripple crest or possibly further offshore, in response to the advection of the vortex ejected at around $162^{\circ}$ in the offshore direction. The same is true of the flow reversal at $0^{\circ}$, except here the sediment cloud appears to be ahead of the corresponding vortex. Similar behaviour is observed in the experiments at flow reversal. This happens because the suspended sediment is redistributed not only by advection but also by diffusion and settling. This may explain as well the differences between the concentration contours predicted by the two models. In fact, the DVPT model does not have any spatial variation in diffusion, nor is there any substantial source of sediment from the bed other than that released at the crest. This is probably why the concentration and vorticity mirror one another in the DVPT model results. The $k-\omega$ model, on the other hand, which does include entrainment of sediment along the entire ripple surface, may have too much variation in diffusion.

Finally, we investigate how each model compares with the experimental concentration plots, shown in the middle panels of Fig. 8. The models are capable of reproducing the time-dependent concentration field reasonably well, both qualitatively and to some extent quantitatively. By analogy with the vorticity dynamics, a suspended sediment cloud develops on the onshore (right-hand) side of the ripple during the onshore flow half cycle. This suspended sediment cloud is ejected into the flow near the on-offshore flow reversal. During the offshore flow half cycle, a 

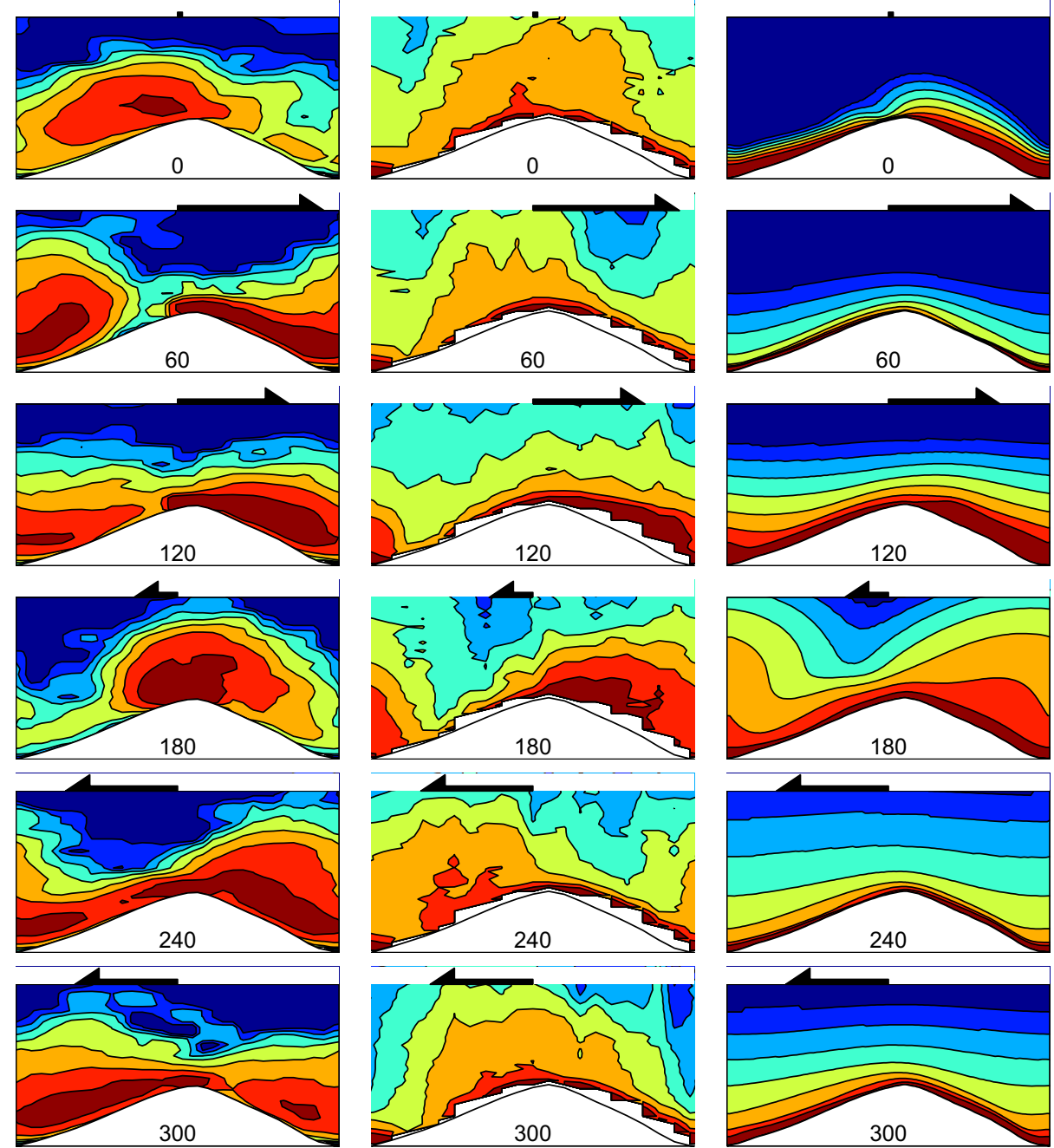

DVPT

DATA

$k-\omega$

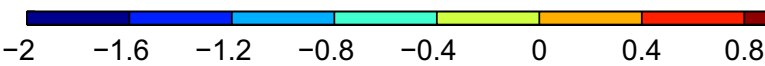

Fig. 8. Suspended sediment concentration contours $\left[\log _{10}(c)\right]$ predicted by the DVPT model (left panels), measured in the experiment (middle panels), and predicted by the $k-\omega$ model (right panels) for the phases shown (deg). The arrows are proportional to the strength of the free-stream velocity.

suspended sediment cloud is generated on the offshore side of the ripple, which is then ejected into the flow near the off-onshore flow reversal. The suspended sediment cloud ejected near the on-offshore flow reversal $\left(\sigma t=162^{\circ}\right)$ is larger and contains more sediment than the suspended sediment cloud ejected around the off-onshore flow reversal $\left(\sigma t=0^{\circ}\right)$. This is due to the flow asymmetry: the onshore velocities are stronger than the offshore velocities. This asymmetry seems to be well represented by the $k-\omega$ model, but tends to be underpredicted by the DVPT model. This is as expected since the diffusion in the DVPT model is not velocity dependent, unlike in the $k-\omega$ model, and the vorticity asymmetry is underpredicted.

Along the ripple surface the $k-\omega$ model is better able to represent the concentration than the DVPT model because of a better near-bed turbulence description. However, as we already observed in Fig. 5, the vortices are not lifted high enough up into the water column in the $k-\omega$ model, and this is reflected in the suspended sediment dynamics (closest agreement between model and data occur at the same phases, $120^{\circ}$ and $180^{\circ}$, for both the vorticity and concentration). The model produces sediment clouds that are very close to the bed at phase $\sigma t=0^{\circ}$, whereas the measurements show considerable amounts of sediment in suspension (as a result of the clouds generated by vortex shedding) that have not settled to the bed completely. However at $\sigma t=120^{\circ}$, and especially at $\sigma t=180^{\circ}$, even though vorticity is underpredicted by the $k-\omega$ model, sediment diffusion appears to be sufficient to produce concentration contours that are comparable to the measured ones. This may suggest that diffusion in the $k-\omega$ model is possibly larger than it should be, as pointed out previously. However, this is not the case for the remaining phases depicted in Fig. $5\left(\sigma t=0^{\circ}, 60^{\circ}, 240^{\circ}\right.$ and $\left.300^{\circ}\right)$, 
when the sediment diffusion is not large enough to compensate for the underprediction of vorticity. It is difficult to test whether the amount of diffusion in the $k-\omega$ model is appropriate in this case for two reasons. Firstly, Reynolds stresses, on which diffusion is scaled, cannot be reliably obtained from the experimental data because of the limited number of flow cycles and the resolution of the velocity measurements. Secondly, the vorticity is not being predicted correctly by the $k-\omega$ model: one would expect the distribution of Reynolds stresses to mirror the distribution of vorticity (Sato, 1987; Perrier, 1996).

With the exception of the case when $\sigma t=180^{\circ}$ (discussed earlier), the DVPT model shows better agreement with the measurements than the $k-\omega$ model in terms of the positions and size of the suspension clouds (higher concentrations at higher elevations above the bed). However, it seems that the DVPT model does not include enough diffusion, especially higher above the rippled bed. As a result, the concentrations within the ejected vortices are overpredicted and the concentrations outside the ejected vortices are underpredicted.

\subsection{Cycle- and ripple-averaged suspended sediment concentrations}

Fig. 9 shows the measured (by the ABS and TSS) and predicted vertical profile of the cycle- and ripple-averaged concentrations. The ABS-measured concentrations below the ripple crest level are noisy compared to those above because of the comparatively small number of non-zero measured concentration values contributing to the hor- izontal average. It can be seen that the ABS and TSS measurements are in good agreement with one another.

In general there is reasonable agreement between the measured and predicted concentration profiles. The $k-\omega$ model prediction and the data show that the cycle- and ripple-averaged concentrations decay exponentially, as expected (see Nielsen, 1992). Close to the ripple crest $(0.19<y /$ $\lambda<0.3)$ the DVPT model slightly overpredicts the concentration (prediction is within a factor of 1.5) and the $k-\omega$ model underpredicts the concentration (prediction is within a factor of 3$)$. Below the ripple crest $(0<y / \lambda<0.19)$ the DVPT model predicts the concentration better than the $k-\omega$ model. This is partly because the DVPT model tends to produce the vortex positions and corresponding concentration contours that are closer to those observed as already discussed, and partly because the $k-\omega$ model produces concentrations that are very large right along the ripple surface. In the case of the DVPT model, the local over- and underpredicted concentrations described in Fig. 8 for the DVPT model compensate for one another to produce a reasonable cycle- and ripple-averaged concentration. Above $y / \lambda=0.35$ both models underpredict the concentration, but by $y / \lambda=0.43$ the $k-\omega$ predicts the larger concentration. In this upper region the $k-\omega$ model produces the more reasonable concentration probably because of its better representation of background turbulence in the outer flow.

\subsection{Net suspended sediment fluxes and transport rates}

The time-varying velocity and ABS concentration measurements can be combined to produce time-varying

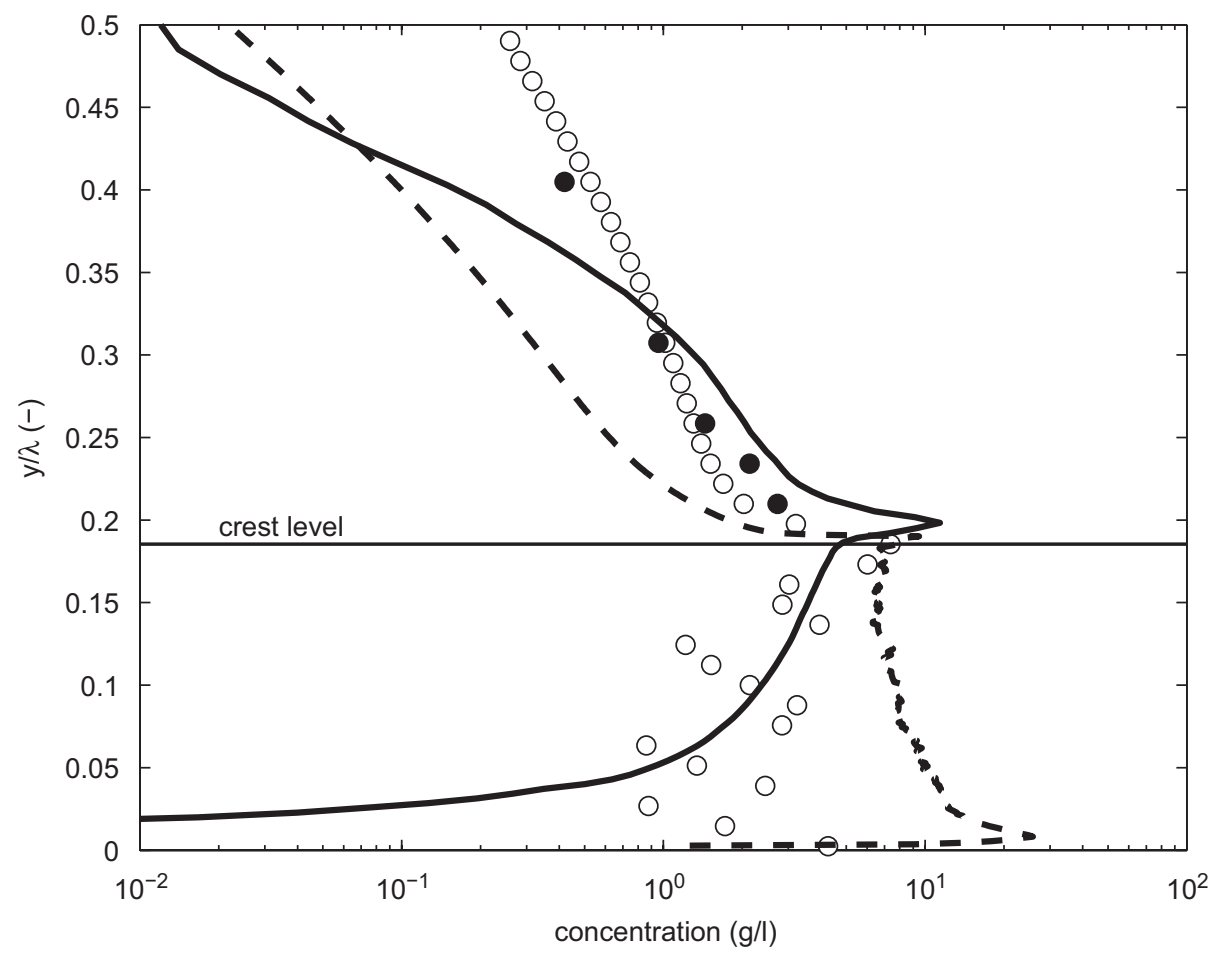

Fig. 9. Cycle- and ripple-averaged suspended sediment concentrations predicted by the DVPT (solid line) and $k-\omega$ (dashed line) models, and measured in the experiment using ABS (open circles) and TSS (closed circles). 
suspended sediment fluxes. The net (cycle-averaged) suspended sediment flux has a wave- and current-related component (defined by $\langle\overline{u c}\rangle-\langle\bar{u} \bar{c}\rangle$ and $\langle\bar{u} \bar{c}\rangle$, respectively, where the overbar denotes a cycle average and the angled brackets denote a ripple average, see also Van der Werf et al., 2007). Fig. 10 shows comparisons between the measured and predicted current-related, wave-related and total cycle-averaged, ripple-averaged suspended sediment fluxes, respectively. Again the measured fluxes below the ripple crest level are noisy compared to those above the crest because of the comparatively small number of nonzero measured flux values contributing to the horizontal average.

Both the DVPT and $k-\omega$ models accurately predict the current-related suspended sediment flux above the ripple crest level. Below this level the DVPT model underestimates and the $k-\omega$ model slightly overestimates the current-related flux. The current-related flux is in the offshore direction due to the relatively strong offshore mean flow in combination with the relatively high sediment concentrations above the onshore (right-hand side) ripple flank (see Figs. 6 and 8). Due to the phase lags between the near-bed peak flow velocities and suspended sediment concentrations related to the vortex ejection at times of flow reversal (see Figs. 5 and 8), the wave-related suspended sediment flux is offshore as well. This process is represented by both models. The $k-\omega$ model systematically underpredicts the wave-related flux above the ripple crest level and consequently the total cycle-averaged flux. The DVPT model does better, except close to the ripple's crest, where it predicts a large onshore wave-related flux peak. The latter is related to the fact that the DVPT model releases the majority of its sediment particles near the crest at a point where the velocity is already overpredicted. Thus, because the velocities are stronger and, consequently, the sediment particle 'masses' are larger for the onshore flow, the result is an onshore near-crest peak in the wave-related and total fluxes. This may indicate the need for a more diffuse injection point of particles near the crest, together with a better representation of the boundary layer.

If we vertically integrate these ripple-averaged suspended sediment fluxes from the ripple trough upwards, we get the corresponding suspended sediment transport rates (see Table 1). Again we distinguish between the wave-related, current-related and total cycle-averaged suspended transport rate. The models predict an 'offshore' (negative) net suspended sediment transport, i.e. against the orbital flow asymmetry, as seen in the data. The $k-\omega$ model in particular predicts the value of the net suspended transport well. Both the wave- and current-related suspended transport rates are offshore and the current-related component dominates over the wave-related component. The $k-\omega$ model underpredicts the wave-related transport component, which is partly the result of an underprediction of the height to which the suspended sediment clouds are lifted (see Figs. 5 and 8). The values of the net suspended transport rate predicted by the DVPT model are lower than
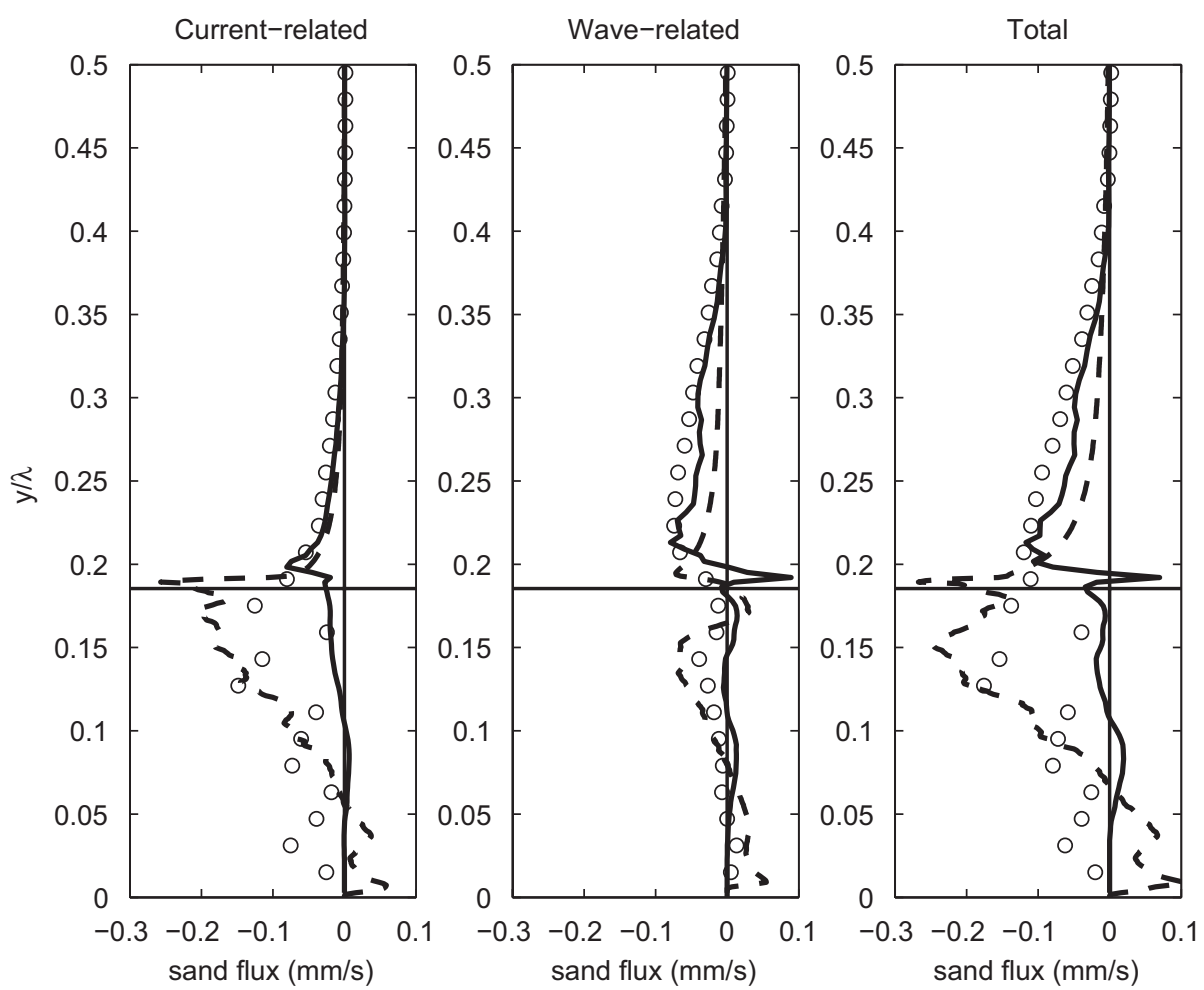

Fig. 10. Current-related, wave-related and total cycle-averaged, ripple-averaged suspended sediment fluxes predicted by the DVPT model (solid lines) and the $k-\omega$ model (dashed lines) and measured in the experiment (open circles). The horizontal (solid) line at $y / \lambda=0.19$ denotes the ripple crest level. 
Table 1

Measured and predicted current-related $\left\langle q_{\mathrm{sc}}\right\rangle$, wave-related $\left\langle q_{\mathrm{sw}}\right\rangle$ and total cycle-averaged $\left\langle q_{\mathrm{s}}\right\rangle$ suspended transport rates in $\mathrm{mm}^{2} / \mathrm{s}$

\begin{tabular}{lccr}
\hline & $\left\langle q_{\mathrm{sc}}\right\rangle$ & $\left\langle q_{\mathrm{sw}}\right\rangle$ & $\left\langle q_{\mathrm{s}}\right\rangle$ \\
\hline DVPT model & -1.6 & -2.3 & -3.9 \\
$K-\omega$ model & -6.6 & -2.2 & -8.8 \\
Data $^{\mathrm{a}}$ & -7.3 & -4.6 & -11.9 \\
\hline
\end{tabular}

${ }^{\mathrm{a}}$ These values differ from those quoted in Van der Werf et al. (2007) because integration is performed down to the ripple surface rather than two grid cells above it.

that of the data. This is caused by a lack of asymmetry in the velocity and suspended sediment concentration during onshore and offshore flow (see Figs. 5 and 8).

It is well known that the total transport rate in general and the bedload transport rate along the ripple surface in particular are responsible for ripple migration (Traykovski et al., 1999; Hoekstra et al., 2004). However in this experiment, in the ripple-averaged sense, it is clearest to define the cycle-averaged total transport rate $\left\langle q_{\mathrm{t}}\right\rangle$ as simply

$\left\langle q_{\mathrm{t}}\right\rangle=\left\langle q_{\mathrm{s}}\right\rangle+\left\langle q_{\mathrm{r}}\right\rangle$,

where $\left\langle q_{\mathrm{s}}\right\rangle$ is the net transport contribution from the cycle- and ripple-averaged suspended sediment, as defined and discussed above, and $\left\langle q_{\mathrm{r}}\right\rangle$ is the transport contribution from the migration of the ripple itself. During the experiment, the ripples migrated onshore with a fairly constant rate of $15-20 \mathrm{~mm} / \mathrm{min}$. If we assume that the ripple shape, as depicted in Fig. 2, remains constant while it migrates, and that there is no transport below the rippletrough level, $\left\langle q_{\mathrm{r}}\right\rangle$ can be estimated by

$\left\langle q_{\mathrm{r}}\right\rangle=c_{\mathrm{r}} f\left(1-\varepsilon_{\mathrm{p}}\right) \eta$,

where $c_{\mathrm{r}}$ is the ripple-migration rate, $f$ is the dimensionless shape factor (given by the area under the curve shown in Fig. 3 multiplied by $1 /(\lambda \eta))$ and $\varepsilon_{\mathrm{p}}(\approx 0.4)$ is the bed porosity. Since $f$ is 0.507 in this case, we find that $\left\langle q_{\mathrm{r}}\right\rangle=6.9 \pm 1.0 \mathrm{~mm}^{2} / \mathrm{s}$. The total net sediment transport, measured directly from mass conservation (see Section 3.2), is $\left\langle q_{\mathrm{t}}\right\rangle=-3.7 \pm 0.7 \mathrm{~mm}^{2} / \mathrm{s}$, so the contribution of the cycle- and ripple-averaged suspended sediment may be deduced from Eq. (4) to be $\left\langle q_{\mathrm{s}}\right\rangle=-10.6 \pm 1.7 \mathrm{~mm}^{2} / \mathrm{s}$. It can be seen that this value of $\left\langle q_{\mathrm{s}}\right\rangle$ agrees closely with that obtained previously from the integration of flux profiles $\left(-11.9 \mathrm{~mm}^{2} / \mathrm{s}\right)$ considering the uncertainties in the measurements. For experiment Mr5b63, the total sediment transport is offshore as a result of the greater offshore suspended transport relative to the smaller onshore ripplemigration transport. Bearing in mind the grain size in suspension (Section 3.4.2), this implies that grains larger than $0.295 \mathrm{~mm}$ are being transported onshore while grains smaller than $0.295 \mathrm{~mm}$ are being transported offshore. While the suspended transport in this case has proved to be the most significant component, this discussion highlights the need for models to be able to predict migration rates as well.

\section{Conclusions}

We have tested two different 2DV sediment transport models against detailed velocity and sediment concentration measurements above full-scale mobile ripples in regular asymmetric oscillatory flow. The models considered are the $k-\omega$ turbulence-closure model of Guizien (2005) and the DVPT model of Malarkey and Davies (2002) and Magar and Davies (2005). This is the first time that such a detailed model intercomparison has been carried out.

A procedure to remove the settling velocity effect from the velocity data was successfully applied. The measured velocity field was first forced to be periodic in the horizontal direction and then the ripple-averaged vertical velocity at each height was removed from the data, because this quantity must be zero at all heights to satisfy continuity. This procedure was necessary since the measured velocities are those of the suspended sediment rather than the water itself. The resulting flow field satisfies better the condition of local tangency close to the rippled bed and the values of the removed ripple-averaged vertical velocities appear to agree reasonably well with the computed settling velocity of suspended sediment.

The models and the data demonstrate that the timedependent velocity and suspended sediment concentration above rippled beds are dominated by the generation of leeside vortices and their subsequent ejection at flow reversal. In general, the flow dynamics are reasonably well predicted by the models. The DVPT model predicts the horizontal and vertical positions and strengths of the vortices at all phases reasonably well, but tends to overpredict the velocity close to the ripple surface because it does not represent the turbulent boundary layer characteristics properly. The $k-\omega$ model, on the other hand, underpredicts the height reached by the vortices, and most of the vorticity tends to occur close to the ripple surface. However, this model is better able to predict the velocity close to the bed. The $k-\omega$ model's underprediction of the heights to which vorticity is lifted is thought to be related to the boundary condition on the vorticity at the ripple surface, which is sensitive to the ripple steepness. The quantitative agreement between the measured and predicted cycle- and ripple-averaged horizontal velocity is reasonable. Both models reproduce the measured offshore flow close to and below the ripple and the DVPT model reproduces the measured onshore flow higher up.

The DVPT model reproduces the horizontal and vertical extent of the growing and ejected sediment clouds reasonably well. However, it overpredicts the concentrations within the vortices and underpredicts the concentrations outside the vortices. This may be because sediment diffusion is not large enough in the DVPT model, and also because it is uniform across the field. The $k-\omega$ model underpredicts the height to which the suspended sediment 
clouds are lifted, which is the result of the vortices not being lifted high enough up into the water column. However, in the $k-\omega$ model the diffusion is possibly larger than it should be, but this is difficult to test. It is important to note here that the main shortcoming of the $k-\omega$ model, and of Reynolds-averaged Navier-Stokes models in general, is that it is difficult to obtain the right balance between vorticity and diffusion (Chang and Scotti, 2004). This depends on the turbulence-closure condition, and the difficulties in calibrating the parameters in it. The measured and predicted cycle- and ripple-averaged suspended sediment concentrations are in reasonable agreement and demonstrate a region of exponential decay. The concentrations are better predicted by the DVPT model in the vicinity of the growing and ejected vortices and by the $k-\omega$ model higher up in the water column and along the ripple surface.

The models are able to reproduce the observed 'offshore' cycle- and ripple-averaged current- and wave-related suspended sediment fluxes from the ripple troughs upwards. As a result, the predicted and measured net suspended sediment transport rates are also offshore and are in reasonable agreement with each other. The measured net suspended transport based on flux measurements was found to be consistent with the net suspended transport deduced from the difference between the measured total transport and the transport associated with the measured ripple migration. For the particular experiment considered in this paper, the net offshore suspended transport, which is the result of only a small fraction $(18 \%)$ of the grain population of the bed, is greater than the net onshore transport due to ripple migration, which involves the entire grain population. However, other conditions of flow and sediment size may result in a net onshore rather than offshore transport, and numerical models, including the 2DV-type models considered here, need to account for ripple migration if they are to properly capture the overall transport process.

\section{Acknowledgements}

This work has been carried out within the SANDPIT project ('Sand Transport and Morphology of Offshore Sand Mining Pits') funded by the European Union (EVK32001-00056) and within the SANTOSS project ('Sand Transport in Oscillatory flows in the Sheet-flow regime') funded jointly by EPSRC in the UK (GR/T28089/01) and STW in the Netherlands (TCB.6586). Vanesa Magar was also partly funded by an EPSRC academic fellowship.
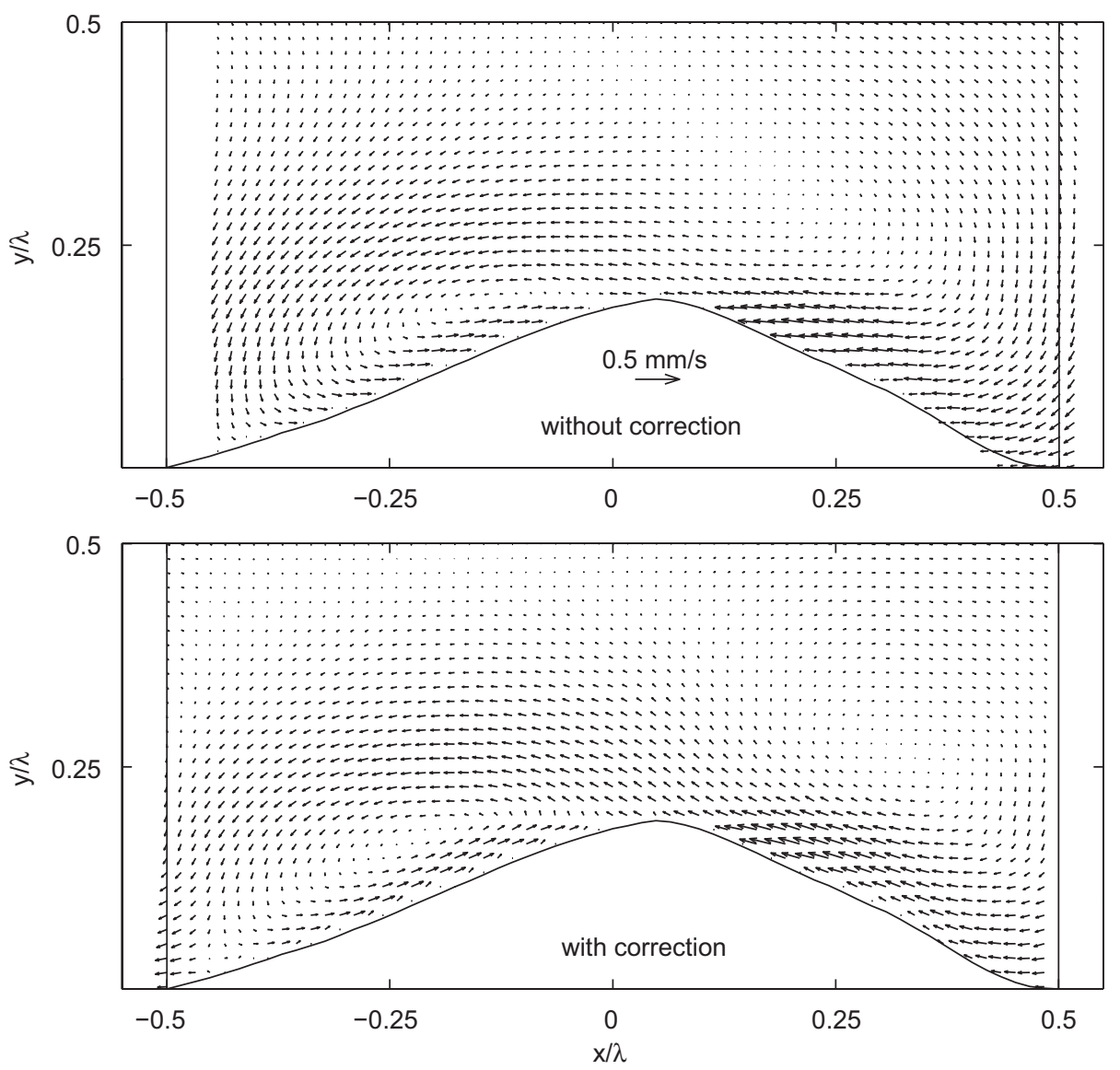

Fig. A1. The effect of forcing periodicity in the ripple wavelength and removing the horizontally averaged vertical velocity on the cycle-mean velocity field (vertical lines correspond to where periodicity is forced and the common velocity scale is shown in the top panel). 


\section{Appendix A. Modification of PIV data}

This appendix explains how the PIV data were modified in order to correct for the settling velocity effect of the sediment, and then demonstrates the difference the modification makes.

The modifications made to the PIV data were as follows.

1. Periodicity was forced at the two ripple troughs:

$\mathbf{u}(-0.5 \lambda, y)=\mathbf{u}(0.5 \lambda, y)$,

2. In anticipation of the vorticity calculation, the PIV velocity data were first interpolated onto a grid such that an integer number of grid cells corresponded to a ripple wavelength $(\Delta x=6.6129 \mathrm{~mm}$ as opposed to $6.4 \mathrm{~mm}$ such that $\lambda=62 \Delta x$ but $\Delta y$ was still $6.4 \mathrm{~mm}$ ).

3. A ripple average of the vertical velocity was taken at each height and each time instant and then removed from the data (since the ripple-averaged vertical velocity must be zero in order to satisfy continuity).

The effect of these changes on the cycle-mean velocity vectors is shown in Fig. A1 (the 'with correction' panel is the same velocity field as that depicted in Fig. 6, except that here every point is shown). It can be seen that the procedure improves the velocity field estimates, especially near the ripple surface such that the vectors satisfy better the condition of local tangency close to the ripple. It can also be seen that the forcing of periodicity is useful because it helps to fill in the gaps in the data, using linear interpolation.

\section{Appendix B. Calculation of vorticity and its consistency}

This appendix explains how the vorticity is calculated from the modified PIV data and then examines its consistency.

The vorticity, $\Omega$, can be readily calculated from the corrected velocity field:

$\Omega=\frac{\Delta u}{\Delta y}-\frac{\Delta v}{\Delta x}$.

However, in order to produce the vorticity contours, it is necessary to use a running-mean or convolution process to reduce the noise in the signal and smooth $\Omega$.

The consistency of the vorticity data is examined here by considering two phases in the wave cycle and showing the effect of increasing the number of cycles over which the data are phase-ensembled. The two phases chosen $\left(0^{\circ}\right.$ and $\left.180^{\circ}\right)$ are depicted in Fig. A2 for phase-ensembling over 1, 2, 3, 4, and 5 wave cycles. Also depicted is the standard deviation of the individual realisations relative to the phase-ensemble.

In the $0^{\circ}$ case, it is clear that the basic structure of the strongest vorticity in the field, around the newly ejected vortex $F_{0}$, is preserved very well through successive ensembles. Also preserved through the phase-ensembling, though not as well, is the old onshore vortex, $N_{0}$. However, in the regions of lower vorticity no discernable structure is preserved. From the standard deviation panel it can be seen
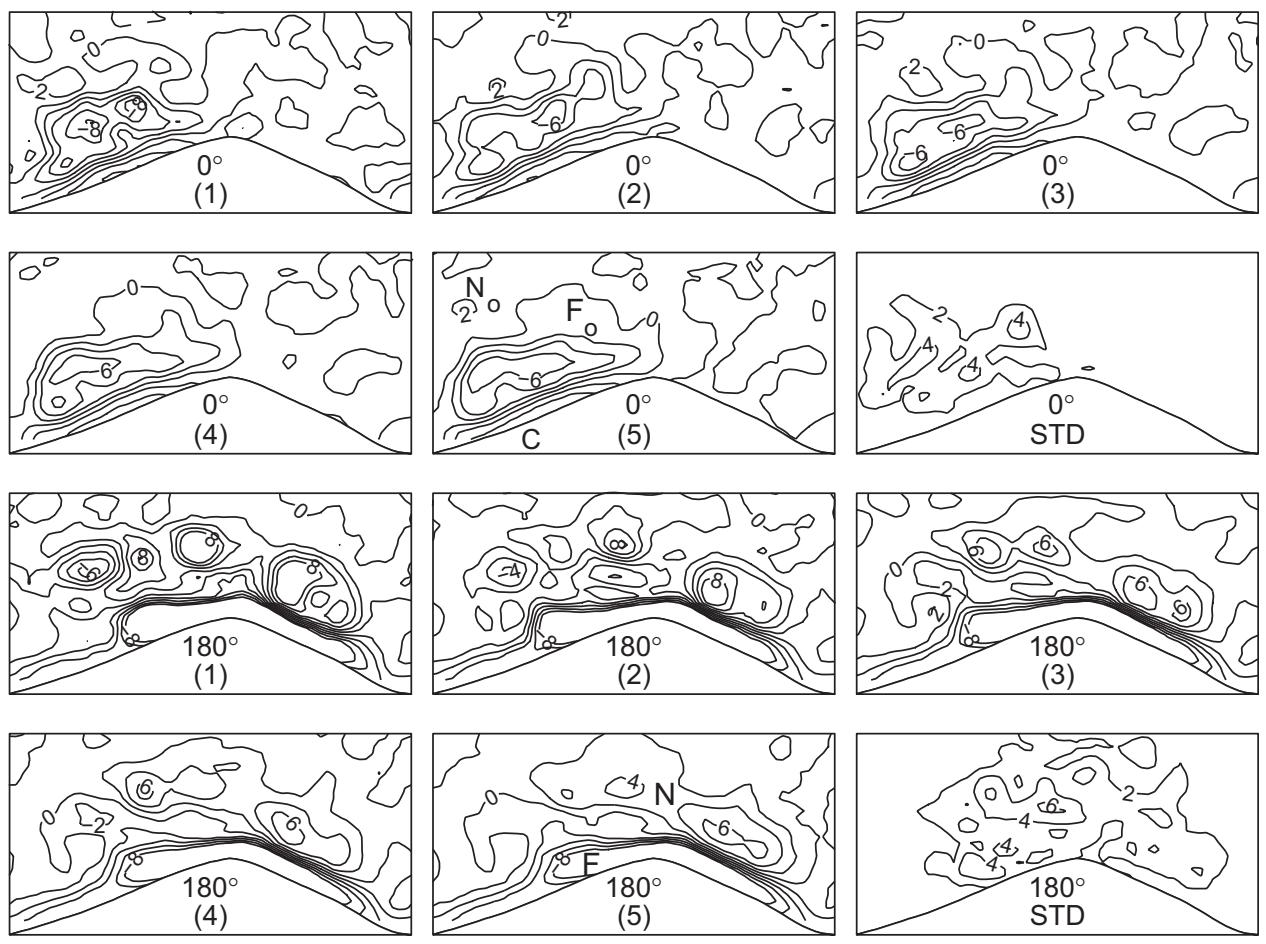

Fig. A2. Non-dimensional vorticity contours $\left(\Omega \lambda / U_{1}\right)$ at the labelled phases for phase-ensembling over different numbers of wave cycles (number in brackets) and standard deviation of the five realisations. 
that only the preserved features have a standard deviation that is smaller in magnitude than the feature itself. In the $180^{\circ}$ case, the structure of the growing vortex, $F$, is again well preserved but the structure of the newly ejected vortex $N$ is not preserved as well, as reflected by the larger and more scattered standard deviation. One initial feature that is lost to the phase-ensembling altogether is the negative vorticity that appears to the left of vortex $N$. The fact that this feature decreases in strength consistently and finally disappears suggests that it is probably spurious. It is clear that while phase-ensembling over a larger number of cycles would probably better capture the vorticity structure, particularly in the $180^{\circ}$ case, the most important features of the flow are preserved.

\section{References}

Andersen, K.H., 1999. Ripples beneath surface waves and topics in shell models of turbulence. Ph.D. Thesis, University of Copenhagen, Denmark.

Bagnold, R.A., 1946. Motion of waves in shallow water: interaction between waves and sand bottoms. Proceedings of the Royal Society of London Series A 187, 1-15.

Blondeaux, P., Scandura, P., Vittori, G., 2004. Coherent structures in an oscillatory separated flow: numerical experiments. Journal of Fluid Mechanics 518, 215-229.

Bosman, J.J., 1982. Concentration measurements under oscillatory motion, Report M1695, Part II. Delft Hydraulics, The Netherlands.

Chang, Y.S., Scotti, A., 2003. Entrainment and suspension of sediments into a turbulent flow over ripples. Journal of Turbulence 4 (019).

Chang, Y.S., Scotti, A., 2004. Modelling unsteady turbulent flow over ripples: Reynolds-averaged Navier-Stokes equations (RANS) versus large eddy simulation (LES). Journal of Geophysical Research 109 (C09012).

Chang, Y.S., Scotti, A., 2006. Turbulent convection of suspended sediments due to flow reversal. Journal of Geophysical Research 111 (C7).

Christiansen, J.P., 1973. Numerical simulation of hydrodynamics by the method of point vortices. Journal of Computational Physics 13, 363-379.

Clubb, G.S., 2001. Experimental study of vortex ripples in full-scale sinusoidal and asymmetric flows. Ph.D. Thesis, Aberdeen University, UK.

Davies, A.G., Thorne, P.D., 2005. Modeling and measurement of sediment transport by waves in the vortex ripple regime. Journal of Geophysical Research 110 (C5).

Doering, J.C., Baryla, A.J., 2002. An investigation of the velocity field under regular and irregular waves over a sand beach. Coastal Engineering 44, 275-300.

Earnshaw, H.C., Greated, C.A., 1998. Dynamics of ripple bed vortices. Experiments in Fluids 25, 265-275.

Eidsvik, K.J., 2006. Large scale modelling of oscillatory flows over a rippled bottom. Continental Shelf Research 26, 318-337.

Engelund, F., Fredsøe, J., 1976. A sediment transport model for straight alluvial channels. Nordic Hydology 7, 293-306.

Faraci, C., 2001. Geometry, migration and evolution of sea ripples. Ph.D. Thesis, University of Catania, Italy.

Faraci, C., Foti, E., 2002. Geometry, migration and evolution of smallscale bedforms generated by regular and irregular waves. Coastal Engineering 47, 35-52.

Fredsøe, J., 1984. Turbulent boundary layer in wave-current motion. Journal of Hydraulic Engineering 110, 1103-1120.

Fredsøe, J., Deigaard, R., 1992. Mechanics of Coastal Sediment Transport. World Scientific, Singapore.

Fredsøe, J., Andersen, K.H., Summer, B.M., 1999. Wave plus current over a ripple-covered bed. Coastal Engineering 38, 177-221.
Grasmeijer, B.T., Kleinhans, M.G., 2004. Observed and predicted bed forms and their effect on suspended sand concentrations. Coastal Engineering 51, 351-371.

Green, M.O., Black, K.P., 1999. Suspended-sediment reference concentration under waves: field observations and critical analysis of two predictive models. Coastal Engineering 38, 115-141.

Guizien, K., 2005. Equilibrium bottom roughness of ripples derived from a 2DHV ripple model. In: Van Rijn, L.C., Soulsby, R.L., Hoekstra, P., Davies, A.G. (Eds.), SANDPIT, Sand Transport and Morphology of Offshore Sand Mining Pits. Aqua Publications, The Netherlands, pp. AB 1-AB 9.

Guizien, K., Dohmen-Janssen, C.M., Vittori, G., 2003. 1DV bottom boundary layer modeling under combined wave and current: turbulent separation and phase lag effects. Journal of Geophysical Research 108 (C1).

Hallermeier, R.J., 1981. Terminal settling velocity of commonly occuring sand grains. Sedimentology 28, 859-865.

Hanes, D.M., Alymov, A., Chang, Y.S., Jette, C., 2001. Wave-formed sand ripples at Duck, North Carolina. Journal of Geophysical Research 108 (C1).

Hansen, E.A., Fredsøe, J., Deigaard, R., 1994. Distribution of suspended sediment over wave-generated ripples. Journal of Waterway Port Coastal and Ocean Engineering 120, 37-55.

Hoekstra, P., Bell, P., Van Santen, P., Roode, N., Levoy, F., Whitehouse, T., 2004. Bedform migration and bedload transport on an intertidal shoal. Continental Shelf Research 24, 1249-1269.

Holmedal, L.E., Myrhaug, D., 2006. Boundary layer flow and net sediment transport beneath asymmetric waves. Continental Shelf Research 26, 252-268.

Longuet-Higgins, M.S., 1981. Oscillating flow over steep sand ripples. Journal of Fluid Mechanics 107, 1-35.

Magar, V., Davies, A.G., 2005. Suspended sediment dynamics over rippled beds in oscillatory flows based on a 2DHV discrete-vortex/ particle-tracking model. In: Van Rijn, L.C., Soulsby, R.L., Hoekstra, P., Davies, A.G. (Eds.), SANDPIT, Sand Transport and Morphology of Offshore Sand Mining Pits. Aqua Publications, The Netherlands, pp. AG 1-AG 9.

Magar, V., Van der Werf, J.J., Eidsvik, K.J., Guizien, K., Malarkey, J., Doucette, J.S., 2005. Comparison between 2DV sediment transport models and measurements of suspended sediment dynamics over fullscale ripples in oscillatory flows. In: Van Rijn, L.C., Soulsby, R.L., Hoekstra, P., Davies, A.G. (Eds.), SANDPIT, Sand Transport and Morphology of Offshore Sand Mining Pits. Aqua Publications, The Netherlands, pp. AH 1-AH 13.

Malarkey, J., Davies, A.G., 2002. Discrete vortex modelling of oscillatory flows over ripples. Applied Ocean Research 24, 127-145.

Malarkey, J., Davies, A.G., Li, Z., 2003. A simple model of unsteady sheet flow sediment transport. Coastal Engineering 48, 171-188.

Marin, F., 2004. Eddy viscosity and Eulerian drift over rippled beds in waves. Coastal Engineering 50, 139-159.

Nielsen, P., 1992. Coastal Bottom Boundary Layers and Sand Transport. World Scientific, Singapore.

Perrier, G., 1996. Numerical modelling of the flow and sand transport by waves and currents over a rippled bed. Ph.D. Thesis, Orsay University, France.

Ribberink, J.S., Al-Salem, A.A., 1994. Sediment transport in oscillatory boundary layers in case of rippled beds and sheet flow. Journal of Geophysical Research 99 (C6), 12,707-12,727.

Saffman, P.G., 1970. A model for inhomogeneous turbulent flow. Proceedings of the Royal Society of London Series A 317, 417-433.

Sato, S., 1987. Oscillatory boundary flow and sand movement over ripples. Ph.D. Thesis, University of Tokyo, Japan.

Scandura, P., Vittori, G., Blondeaux, P., 2000. Three-dimensional oscillatory flow over steep ripples. Journal of Fluid Mechanics 412, 355-378.

Sleath, J.F.A., 1984. Seabed Mechanics. Wiley, NewYork.

Thorne, P.D., Williams, J.J., Davies, A.G., 2002. Suspended sediment under waves measured in a large-scale flume facility. Journal of Geophysical Research 107 (C8). 
Thorne, P.D., Davies, A.G., Williams, J.S., 2003. Measurements of nearbed intra-wave sand entrainment above vortex ripples. Geophysical Research Letters 30 (20), 2028.

Traykovski, P., Hay, A.E., Irish, J.D., Lynch, J.F., 1999. Geometry, migration, and evolution of wave orbital ripples at LEO-15. Journal of Geophysical Research 104 (C1), 1505-1524.

Tseng, Y.H., Ferziger, J.H., 2004. Large-eddy simulation of turbulent wavy boundary flow-illustration of vortex dynamics. Journal of Turbulence 5 (034).

Van der Werf, J.J., 2006. Sand transport over rippled beds in oscillatory flow. Ph.D. Thesis, University of Twente, The Netherlands.

Van der Werf, J.J., Ribberink, J.S., O'Donoghue, T., Doucette, J.S., 2006. Modelling and measurement of sand transport process over full-scale ripples in oscillatory flow. Coastal Engineering 53, 657-673.
Van der Werf, J.J., Doucette, J.S., O’Donoghue, T., Ribberink, J.S., 2007. Detailed measurements of velocities and suspended sand concentrations over full-scale ripples in regular oscillatory flow. Journal of Geophysical Research 112 (F02012).

Vincent, C.E., Green, M.O., 1990. Field measurements of the suspended sand concentration profiles and fluxes and of the resuspension coefficient $\lambda_{0}$ over a rippled bed. Journal of Geophysical Research 95 (C7), 591-601.

Watanabe, Y., Matsumoto, S., Saeki, H., 2003. Three-dimensional boundary layer flow over ripples. Proceedings Coastal Sediments, Clear water Beach, USA (CD-ROM).

Williams, J.J., Bell, P.S., Thorne, P.D., 2003. Field measurements of flow fields and sediment transport above mobile bed forms. Journal of Geophysical Research 108 (C4). 